\title{
MINIMAL GENUS OF LINKS AND FIBERING OF CANONICAL SURFACES
}

\author{
A. STOIMENOW
}

ABstract. This paper contains some further applications of the study of knot diagrams by genus. Introducing a procedure of regularization for knot generators, and using invariants derived from the Jones polynomial (degrees, congruences, and the FiedlerPolyak-Viro Gauß diagram formulas for its Vassiliev invariants), we examine the existence of genus-minimizing diagrams for almost alternating and almost positive knots. In particular, we examine the existence of such knots such that either all or none of their almost alternating/positive diagrams have the minimal genus property. We prove that the genus of almost positive nonsplit links is determined by the Alexander polynomial.

\section{Contents}

1. Introduction . . . . . . . . . . . . . . . . . 400

2. Preliminaries .................. . . . 402

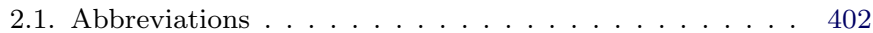

2.2. Knots and knot diagrams . . . . . . . . . . . . . 403

2.3. Rational, pretzel, and Montesinos links . . . . . . . . . . 407

2.4. Link polynomials . . . . . . . . . . . . . . . . 409

2.5. The signature . . . . . . . . . . . . . . . 411

2.6. Gauß sum invariants . . . . . . . . . . . . . . . 411

2.7. Genus generators . . . . . . . . . . . . . . . . . 413

3. Genus-minimizing almost alternating diagrams . . . . . . . . 416

4. Almost positive knots . . . . . . . . . . . . . . . . . . . . . . . 424

4.1. The degree of the Alexander polynomial . . . . . . . . . 424

4.2. General properties of examples . . . . . . . . . . . . . 433

4.3. The Vassiliev invariant test . . . . . . . . . . . . . . . . . 434

4.4. Regularization of generators . . . . . . . . . . . . . 438

4.5. Selection and verification of examples . . . . . . . . . . 441

4.6. Almost special alternating knots and a problem . . . . . 443

Received August 9, 2015; received in final form January 19, 2016.

2010 Mathematics Subject Classification. Primary 57M25. Secondary 57N10, 53D10, $57 \mathrm{M} 15$. 
Acknowledgments . . . . . . . . . . . . . . . . . . 445

References . . . . . . . . . . . . . . . . 445

\section{Introduction}

Introducing the genus $g(K)$ of a knot $K$, Seifert [64] gave a construction of compact oriented surfaces in 3-space bounding the knot (Seifert surface) by an algorithm starting with some diagram of the knot (see [3, §4.3] or [62]). The surface given by this algorithm is called canonical. A natural problem is to determine when the diagram is genus-minimizing (or of minimal genus), that is, its canonical Seifert surface has minimal genus (among all Seifert surfaces of the knot). This problem has been studied over a long period. First, the minimal genus property was shown for alternating diagrams, independently by Crowell [16] and Murasugi [50]. Their algebraic proof uses the Alexander polynomial $\Delta[6]$ and the inequality $\max \operatorname{deg} \Delta \leq g$ (which thus they prove to be exact for alternating knots). Later Gabai [26] developed a geometric method using foliations, called disk decomposition, and showed that this method is successful, too, for alternating diagrams. Murasugi [51] introduced the operation *-product. It was shown to behave naturally with respect to the Alexander polynomial by himself, and later by Gabai [25], [27] in the geometrical context. These results imply the extension of the minimal genus property of alternating diagrams to the homogeneous diagrams of [13]. An important other subclass of the class of homogeneous diagrams and links are the positive diagrams and links. Such links have been considered (in general or in special cases) independently before. (See, e.g., [11], [15], [22], [57], [63], [80], [82], [83].) The minimal genus property for such diagrams follows from yet a different source, the work of Bennequin [8] on contact structures. His inequality (Theorem 3 in that paper) in fact allows one to estimate the difference between the genus of the diagram and the genus of the knot in terms of the number of positive or negative crossings.

Recently other extensions of the class of alternating and positive links have been proposed, in an attempt to generalize the variety of well-known results for them. In [4], Adams et al. introduced almost alternating links and later [2] toroidally alternating links. While some of the topological results for alternating links could be extended, there is a lot of evidence that the useful properties [78], [79], [39], [53], [54] of link polynomials [36], [23], [40] can not.

The idea of considering 'almost'-... diagrams is natural and occurred in the case of almost positive links first independently in papers of Cromwell [13] (as a technical detail) and (with direct focus) Przytycki and Taniyama [61]. Przytycki observed that such links can be studied based on Taniyama's previous work [77], but their manuscript, despite being announced a long ago, was completed only recently. In the meantime, I had studied this class using several combinatorial methods [70], [71] and extended most of the results on 
link polynomials of positive links to almost positive links. With regard to Seifert surfaces, almost alternating (and implicitly almost positive) diagrams were considered recently by Hirasawa [32] and Goda-Hirasawa-Yamamoto [30]. In the latter paper, using cut-and-paste methods, a criterion was given to decide for an almost alternating diagram when its canonical Seifert surface is a fiber surface. Later, an extended and simpler form of this criterion was found, also with a simpler combinatorial proof [71]. The work of [71] also implied a criterion to decide when the surface is of minimal genus. Here we will use this result and several types of knot invariants to investigate the question whether some, or all, almost positive or almost alternating link diagrams $D$ of a particular link $L$ give a minimal genus surface. Let us write (see Section 2.2) $\chi(L)$ and $\chi(D)$ for the Euler characteristic of $L$ and $D$, resp. (Thus, $D$ has minimal genus if and only if $\chi(L)=\chi(D)$.)

THEOREM 1.1. Any almost alternating link $L$ has almost alternating diagrams $D$ whose surfaces have arbitrarily large genus (in particular, not all have minimal genus). That is, $\chi(L)-\chi(D)$ can be arbitrarily large for any almost alternating link $L$.

This is an easy observation based on Adams' tongue move, and is included only for completeness. More effort will be required to show the following results, that considerably extend the three examples $9_{42}, 9_{44}$ and $9_{45}$ in [30]. We start with non-fibered examples, by classifying them into several cases. We will assume knowledge of the standard facts [62] that if a knot $K$ is fibered, then maxdeg $\Delta(K)=g(K)$, max cf $\Delta(K)= \pm 1$ (see Section 2.4 for explanation of these notations), and a fiber and minimal genus surface are unique (up to isotopy) and coincide.

THEOREM 1.2. There are almost alternating knots with $\Delta=1$, none of whose almost alternating diagrams has a minimal genus surface. Among them there are knots that

(a) have no diagrams at all with a minimal genus surface, or

(b) have such diagrams.

There are non-fibered almost alternating knots with minimal genus diagrams, but such that none of these diagrams are almost alternating, whose Alexander polynomials have the following properties:

(c) $\max \operatorname{deg} \Delta=g$, but $\max \operatorname{cf} \Delta \neq \pm 1$, or

(d) $\max \operatorname{deg} \Delta=g$, and $\max \operatorname{cf} \Delta= \pm 1$.

There are infinitely many knots for each of these four combinations of properties.

We obtain fibered examples (as in [30]) as well, but we have in fact the following stronger statement. It generalizes also (even if by totally different means) the result of Morton [48] (to which we will come back shortly). 
THEOREM 1.3. There are infinitely many fibered almost alternating knots with the same Alexander polynomial, whose fibers are canonical surfaces.

(Fibered knots, without examination of almost alternation, whose fibers are not canonical were constructed by Nakamura [56].) An a consequence, we obtain the following corollary.

COROLLARY 1.1. There are infinitely many fibered almost alternating knots with diagrams of minimal genus but no almost alternating such diagrams.

All these results suggest that, at least from the present point of view, almost alternating links are a too large class to inherit many interesting properties.

Turning to almost positive knots, one immediately observes a rather different situation. Theorem 1.1 is no longer true, since, by Bennequin's inequality, we have for an almost positive diagram $D$ of a link $L$,

$$
\chi(D) \in\{\chi(L)-2, \chi(L)\} .
$$

(For a longer discussion see, e.g., [68].) In [71] we found how to decide to which type the diagram $D$ belongs (Lemma 3.1 below), and almost positive links $L$ usually admit diagrams $D$ of both types. We will show here, though, that exceptions occur on either side.

THEOREM 1.4. There exist almost positive knots with either none or all of their almost positive diagrams having minimal genus.

Even if this theorem appears to say much less than the previous ones, it requires more effort than all of them taken together, in the form of a "regularization" procedure for knot generators. (See also the remarks in Section 4.2.) The fact that many of the link polynomial properties of positive links extend to almost positive links, creates considerable difficulties in obtaining and proving the correctness of the corresponding examples. In particular, concerning the Alexander polynomial, we will show the following theorem: ${ }^{1}$

THEOREM 1.5. If $L$ is an almost positive non-split link, then $2 \max \operatorname{deg} \Delta(L)=1-\chi(L)$.

\section{Preliminaries}

2.1. Abbreviations. First, we fix some general (mathematical and linguistic) terminology.

For a set $S$, the expressions $|S|$ and $\# S$ are equivalent and both denote the cardinality of $S$. In the sequel the symbol ' $C$ ' denotes a not necessarily proper inclusion.

\footnotetext{
1 This theorem was first claimed in a preliminary version of [71], but the subtlety of its argument was originally overlooked by the author.
} 


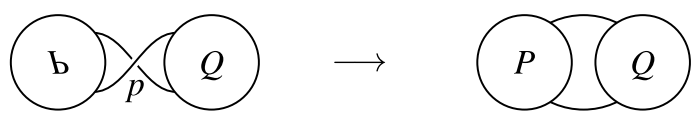

FiguRE 1. Eliminating a reducible crossing.

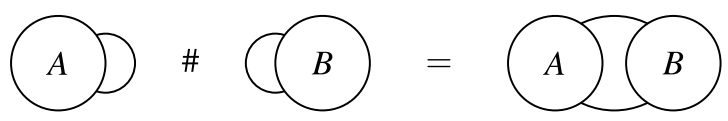

FiguRE 2. Diagram connected sum.

2.2. Knots and knot diagrams. Knots/links and their diagrams must be oriented (even if orientation is not always displayed).

A crossing $p$ in a knot diagram $D$ is called reducible (or nugatory) if it looks like on the left of Figure 1 (or its reflection). The diagram $D$ is called reducible if it has a reducible crossing; otherwise, it is called reduced. The reducing of the reducible crossing $p$ is the move depicted on Figure 1. Each diagram $D$ can be (made) reduced by a finite number of these moves.

We assume in the following all diagrams reduced, unless otherwise stated.

The diagram on the right of Figure 2 is called the connected sum $A \# B$ of the diagrams $A$ and $B$. We say that $A$ and $B$ are (connected sum) factors of $D$. If a diagram $D$ can be represented as the connected sum of diagrams $A$ and $B$, such that both $A$ and $B$ have at least one crossing, then $D$ is called composite; otherwise, it is called prime. A knot or link $K$ is prime if, whenever $D=A \# B$ is a composite diagram of $K$, one of $A$ and $B$ represent an unknotted arc (but not both; the unknot is not prime per convention). Every diagram (and link) has a decomposition into prime factors, unique up to permutation.

THEOREM 2.1 ([46]). If $D$ is a prime alternating non-trivial diagram of a link $K$, then $K$ is prime.

A diagram $D$ is split or disconnected if its plane curve is a disconnected set in $\mathbb{R}^{2}$, that is, there is a simple closed curve disjoint from $D$, whose interior and exterior contain parts of $D$. A diagram which is not disconnected is connected or non-split. A link is a split link if it has a split diagram, and otherwise a non-split link. In analogy to connected sum, one can present every diagram and every link as a split union of split components (which are non-split diagrams/links). A split component of a link (resp., diagram) is trivial, if it is an unknot(ted circle).

The (Seifert) genus $g(K)$ resp. Euler characteristic $\chi(K)$ of a knot or link $K$ is said to be the minimal genus resp. maximal Euler characteristic of a Seifert surface of $K$. For a diagram $D$ of $K, g(D)$ is defined to be the genus 


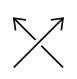

(a)

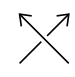

(b)

Figure 3. Positive (a) and negative (b) crossing.

of the Seifert surface obtained by Seifert's algorithm on $D$, and $\chi(D)$ its Euler characteristic. Let $c(D)$ denote the number of crossings of $D$; write $n(D)=$ $n(K)$ for the number of components of $D$ or $K$ (i.e., $n(K)=1$ if $K$ is a knot), and $s(D)$ for the number of Seifert circles of $D$. Then $\chi(D)=s(D)-c(D)$ and $2 g(D)=2-n(D)-\chi(D)$.

TheOREm 2.2 (See [50], [16], [26], [13]). If $D$ is an alternating or positive diagram of $K$, then $g(K)=g(D)$ and $\chi(D)=\chi(K)$.

The crossing number $c(K)$ is the minimal crossing number of all diagrams $D$ of $K$. The canonical genus $\tilde{g}(K)$ resp. canonical Euler characteristic $\tilde{\chi}(K)$ is defined as the minimal genus resp. maximal Euler characteristic of all diagrams of $K$. In general, we can have $g(K)<\tilde{g}(K)$, that is, no diagrams of $K$ of minimal genus (see [49]).

The writhe or sign of a crossing is a number \pm 1 . A crossing as in Figure 3(a) has writhe 1 and is called positive. A crossing as in Figure 3(b) has writhe -1 and is called negative. The writhe $w(D)$ of a link diagram $D$ is the sum of writhes of all its crossings.

Let $c_{ \pm}(D)$ be the number of positive, respectively negative crossings of a diagram $D$, so that $c(D)=c_{+}(D)+c_{-}(D)$ and $w(D)=c_{+}(D)-c_{-}(D)$. Let $c_{ \pm}(K)$ for a knot $K$ denote the minimal number of positive resp. negative crossings of a diagram of $K$.

Definition 2.1. A clasp is a tangle that consists of two crossings, that is, a pair of crossings that bound a bigonal (2-corner) region in the plane complement of a diagram. The clasp is called positive, negative or trivial, if both crossings are positive/negative, resp. of different sign. Depending on the orientation of the involved strands (for an oriented diagram), we distinguish between

a reverse clasp

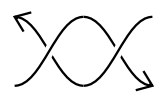
and

a parallel clasp

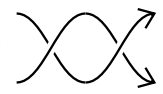

(both up to reflection). Thus, a clasp is reverse if it contains a full Seifert circle, and parallel otherwise.

Definition 2.2. A flype is a move on a diagram shown in Figure 4. We say that a crossing admits a flype if it can be represented as the crossing $p$ displayed in the diagrams in the figure (up to reflection), and both tangles have at least one crossing. 




Figure 4. A flype near the crossing $p$.
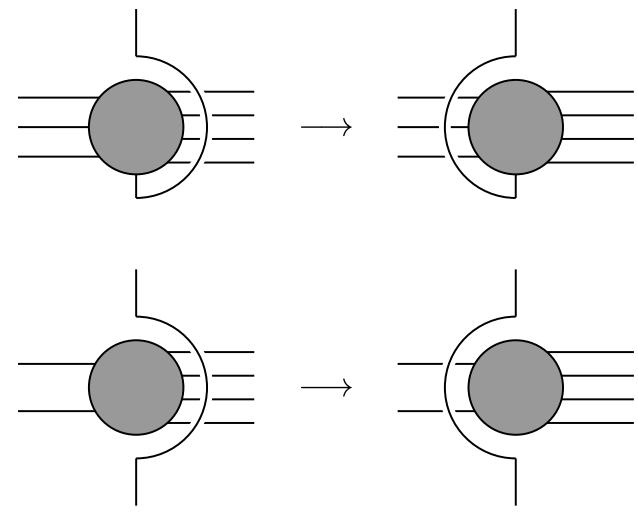

FiguRE 5. Wave-moves. The number of strands on left and right of the shaded circle may vary. It is only important that the parities are equal resp. different, and that the leftoutgoing strands are fewer that the right-outgoing ones.

By the fundamental work of Menasco-Thistlethwaite, we have a proof of the Tait flyping conjecture.

TheOREm 2.3 ([47]). For two alternating diagrams of the same prime alternating link, there is a sequence of flypes taking the one diagram into the other.

A wave move, or bridge rerouting, is a replacement of a too long bridge by a shorter one; see Figure 5 (or [69] for example).

A diagram is almost alternating [3], [4], [2], [30] if it can be turned by one crossing change into an alternating one. A knot is almost alternating, if it has an almost alternating diagram, but is not alternating.

A diagram is positive if all its crossings are positive. A diagram is almost positive if all its crossings are positive except exactly one. A knot is positive if it has a positive diagram. (See, e.g., [15], [57], [82], [83].) It is almost positive if it is not positive but has an almost positive diagram. More generally, a diagram $D$ is $k$-almost positive if it has exactly $k$ negative crossings, that is, 
$c_{-}(D)=k$, and a knot $K$ is $k$-almost positive if it has a $k$-almost positive, but no $k-1$-almost positive diagram, that is, $c_{-}(K)=k$.

A Seifert circle is called separating if both of its complementary regions in the plane contain other Seifert circles. A diagram is special if no Seifert circle is separating. It is an easy observation that for connected diagrams two of the properties alternating, positive/negative and special imply the third. A diagram with these properties is called special alternating. A knot is special alternating if it has a special alternating diagram. Such knots were introduced and studied by Murasugi [53] and have a series of special features. Contrarily, all knots have a special (not necessarily alternating) diagram. Hirasawa [31] shows how to a modify any knot diagram $D$ into a special diagram $D^{\prime}$ so that $g(D)=g\left(D^{\prime}\right)$ (actually, the canonical surfaces of $D$ and $D^{\prime}$ are isotopic).

DEFINITION 2.3. In analogy to almost positive and almost alternating, one can also define a diagram to be almost special alternating if it turns into a special alternating diagram by one crossing change. A knot is almost special alternating if it is not special alternating, but has an almost special alternating diagram.

The notion of a homogeneous diagram/link was introduced by Cromwell [13], in an attempt to extend certain results on positive and alternating links.

Definition 2.4 (See $[13, \S 1]$ ). The Seifert picture (union of Seifert circles) of a link diagram $D$ separates the plane into regions. A non-empty part of $D$ lying in some such region is called a block. Then we say that $D$ is homogeneous if all blocks $D_{i}$ of $D$ are positive or negative (i.e., special alternating). A link is homogeneous if it has a homogeneous diagram.

In the terms of Definition 2.4, a Seifert circle is separating if it bounds blocks on both sides, and a diagram is special if it has only one block. In particular, any of the blocks of $D$ is special.

Since in positive/negative diagrams all blocks are positive/negative, such diagrams are homogeneous. Alternating diagrams are also homogeneous, this time so that blocks which are bordering along a Seifert circle have opposite sign.

Definition 2.5 (See, for example, [55]). The operation that reconstructs a diagram from its blocks by gluing them back along the separating Seifert circles is called (diagrammatic) *-product or Murasugi sum.

This operation is (here) defined for a pair of diagrams $A, B$ and a pair of distinguished non-separating Seifert circle $a, b$. We draw $A$ so that all of it lies in the interior of $a$ and $B$ so that all of $B$ is in the exterior of $b$. Then we identify $a$ and $b$, such that at a place where a crossing is attached to $b$ (resp. $a$ ) we delete an interval of $a$ (resp. $b$ ). There may be a sequence of consecutive deleted intervals along $a$ or $b$; we identify them. Then the number of such deleted intervals is an even integer; it can be called the degree of the Murasugi 
sum. A Murasugi sum of degree 2 is just a connected sum. A Murasugi sum of degree 4 is called a plumbing. If one of $A$ or $B$ is a Hopf band, it is a Hopf plumbing. The converse operations to (Hopf) plumbing and Murasugi sum are called (Hopf) deplumbing and Murasugi desum. (See, e.g., [30] for some geometric pictures.)

The operation on the canonical surfaces of these diagrams is likewise called Murasugi sum. (This operation can be defined for Seifert surfaces in a more general form.)

Definition 2.6. A knot or link $L$ is fibered, if $S^{3} \backslash L$ is a surface bundle over a circle.

TheOREm 2.4 (Neuwirth-Stallings). If $L$ is fibered, then a fiber surface is a minimal genus surface, and a minimal genus surface is unique.

Definition 2.7. A knot or link $L$ will be called canonically fibered, if $L$ is fibered, and the fiber is a canonical surface for some diagram of $L$.

The following helpful theorem of Gabai will be applied a few times.

Theorem 2.5 (Gabai [27], [28], [29]). If a surface $S$ decomposes as Murasugi sum $A * B$, then $S$ is of minimal genus if and only if $A$ and $B$ are so, and $S$ is a fiber surface if and only if $A$ and $B$ are so.

We will use, unless explicitly noted otherwise, KnotScape's [34] notation for $\geq 11$ crossing knots throughout the rest of the paper. It is organized so that non-alternating knots are appended to alternating ones instead of using ' $a$ ' and ' $n$ ' superscripts. For $\leq 10$ crossings, we use the numbering of [62], with the Perko duplication removed (and index of the last four knots shifted down by 1$)$.

The mirror image of a diagram $D$ is written $! D$, and $! K$ is the mirror image of $K$. Clearly $g(! D)=g(D)$ (and therefore $\tilde{g}(K)=\tilde{g}(! K)$ ), and $g(! K)=g(K)$.

2.3. Rational, pretzel, and Montesinos links. We will repeatedly mention (specific) rational, pretzel, and Montesinos tangles, diagrams and links, and thus we clarify here (our convention of) terminology. In the following, we use the approach of Conway [12].

Definition 2.8. A tangle diagram is a diagram consisting of strands crossing each other, and having four ends. A rational tangle diagram is the one that can be obtained from the primitive Conway tangle diagrams by iterated left-associative product in the way displayed in Figure 6. (A simple but typical example of is shown in the figure.)

Let the continued (or iterated) fraction $\left[\left[s_{1}, \ldots, s_{r}\right]\right]$ for integers $s_{i}$ be defined inductively by $[[s]]=s$ and

$$
\left[\left[s_{1}, \ldots, s_{r-1}, s_{r}\right]\right]=s_{r}+\frac{1}{\left[\left[s_{1}, \ldots, s_{r-1}\right]\right]} .
$$



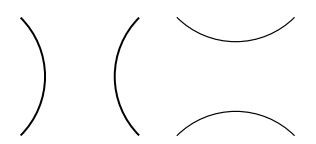

$\infty$

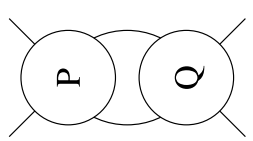

$\operatorname{sum} P, Q$
0

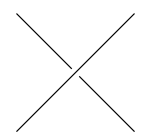

1

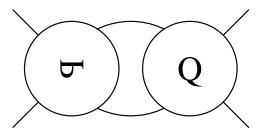

product $P Q$

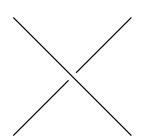

$-1$

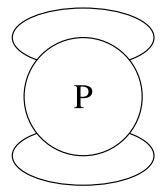

closure $\bar{P}$

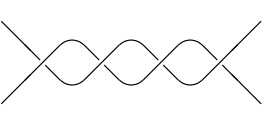

4

Figure 6. Conway's tangles and operations with them. (The designation 'product' is very unlucky, as this operation is neither commutative, nor associative, nor is it distributive with 'sum'. Also, 'sum' is associative, but not commutative.)

The rational tangle $T(p / q)$ is the one with Conway notation $c_{1} c_{2} \ldots c_{n}$, when the $c_{i}$ are chosen so that

$$
\left[\left[c_{1}, c_{2}, c_{3}, \ldots, c_{n}\right]\right]=\frac{p}{q} .
$$

One can assume without loss of generality that $(p, q)=1$, and $0<q<|p|$. A rational (or 2-bridge) link $S(p, q)$ is the closure of $T(p / q)$.

Montesinos links (see, e.g., [44]) are generalizations of pretzel and rational links and special types of arborescent links. They are denoted in the form $M\left(\frac{q_{1}}{p_{1}}, \ldots, \frac{q_{n}}{p_{n}}, e\right)$, where $e, p_{i}, q_{i}$ are integers, $\left(p_{i}, q_{i}\right)=1$ and $0<\left|q_{i}\right|<p_{i}$. Sometimes $e$ is called the integer part, and $n$ the length of the Montesinos link. If $e=0$, it is omitted in the notation.

To visualize the link, let $p_{i} / q_{i}$ be continued fractions of rational tangles $c_{1, i} \ldots c_{n_{i}, i}$ with $\left[\left[c_{1, i}, c_{2, i}, c_{3, i}, \ldots, c_{l_{i}, i}\right]\right]=\frac{p_{i}}{q_{i}}$. Then $M\left(\frac{q_{1}}{p_{1}}, \ldots, \frac{q_{n}}{p_{n}}, e\right)$ is the link that corresponds to the Conway notation

$$
\left(c_{1,1} \ldots c_{l_{1}, 1}\right),\left(c_{1,2} \ldots c_{l_{2}, 2}\right), \ldots,\left(c_{1, n} \ldots c_{l_{n}, n}\right), e 0 .
$$

The defining convention is that all $q_{i}>0$ and if $p_{i}<0$, then the tangle is composed so as to give a non-alternating sum with a tangle with $p_{i \pm 1}>0$. This defines the diagram up to mirroring.

A typical example is shown on Figure 7.

An easy exercise shows that if $q_{i}>0$ resp. $q_{i}<0$, then

$$
M\left(\ldots, q_{i} / p_{i}, \ldots, e\right)=M\left(\ldots,\left(q_{i} \mp p_{i}\right) / p_{i}, \ldots, e \pm 1\right),
$$

that is, both forms represent the same link (up to mirroring). 


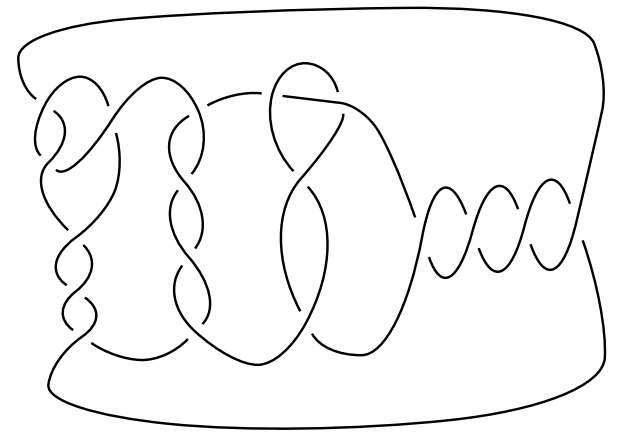

Figure 7 . The Montesinos knot $M(3 / 11,-1 / 4,2 / 5,4)$ with Conway notation $(213,-4,22,40)$.

If the length $n<3$, an easy observation shows that the Montesinos link is in fact a rational link. A pretzel link is a Montesinos link with all $\left|q_{i}\right|=1$. Geometric properties of Montesinos links are discussed in detail in [10].

A Montesinos diagram (resp. rational or pretzel diagram) is the diagram of a closed Montesinos (or rational, or pretzel) tangle. In other words, it is a diagram that can be given in Conway notation (3) in the above specified manner (or its described special cases).

2.4. Link polynomials. Let $X \in \mathbb{Z}\left[t, t^{-1}\right]$. The minimal or maximal degree $\min \operatorname{deg} X$ or $\max \operatorname{deg} X$ is the minimal resp. maximal exponent of $t$ with non-zero coefficient in $X$. Let $\operatorname{span}_{t} X=\max _{\operatorname{deg}} X-\min _{t} \operatorname{deg}_{t} X$. The coefficient in degree $d$ of $t$ in $X$ is denoted $[X]_{t^{d}}$ or $[X]_{d}$. The leading coefficient $\max \operatorname{cf} X$ of $X$ is its coefficient in degree $\max \operatorname{deg} X$. If $X \in \mathbb{Z}\left[x_{1}^{ \pm 1}, x_{2}^{ \pm 1}\right]$, then $\max \operatorname{deg}_{x_{1}} X$ denotes the maximal degree in $x_{1}$. Minimal degree and coefficients are defined similarly, and of course $[X]_{x_{1}^{k}}$ is regarded as a polynomial in $x_{2}^{ \pm 1}$.

The skein polynomial $P$ [23], [43] is a Laurent polynomial in two variables $l$ and $m$ of oriented knots and links and can be defined by being 1 on the unknot and the (skein) relation

$$
l^{-1} P(\nwarrow \nearrow)+l P(\nwarrow)=-m P(\nearrow \nearrow) .
$$

(The convention differs from [43] by the interchange of $l$ and $l^{-1}$.) We will denote in each triple as in (5) the diagrams (from left to right) by $D_{+}, D_{-}$and $D_{0}$. For a diagram $D$ of a link $L$, we will use all of the notations $P(D)=P_{D}=$ $P_{D}(l, m)=P(L)=P_{L}(l, m)$ for its skein polynomial, with the self-suggestive meaning of indices and arguments. 
The Jones polynomial [36] $V$, and (one variable) Alexander polynomial [6] $\Delta$ are obtained from $P$ by the substitutions (with $i=\sqrt{-1}$ )

$$
\begin{aligned}
& V(t)=P\left(-i t, i\left(t^{-1 / 2}-t^{1 / 2}\right)\right), \\
& \Delta(t)=P\left(i, i\left(t^{1 / 2}-t^{-1 / 2}\right)\right) .
\end{aligned}
$$

Hence, these polynomials also satisfy corresponding skein relations. (In algebraic topology, the Alexander polynomial is usually defined only up to units in $\mathbb{Z}\left[t, t^{-1}\right]$; the present normalization is so that $\Delta(t)=\Delta(1 / t)$ and $\Delta(1)=1$. For links there are also multi-variable versions, but we use only the one-variable polynomial throughout the paper.)

We will use sometimes instead of $\Delta$ also the Conway polynomial [12] $\nabla(z)$ with $\nabla\left(t^{1 / 2}-t^{-1 / 2}\right)=\Delta(t)$. It satisfies the skein relation

$$
\nabla\left(D_{+}\right)-\nabla\left(D_{-}\right)=z \nabla\left(D_{0}\right) .
$$

Note that $\max \operatorname{deg} \nabla=2 \max \operatorname{deg} \Delta$, which we use in particular to implicitly restate some of the results of [13] in the sequel. It is well known that for a link $L$ of $n$ components, $\nabla_{L} \in z^{n-1} \mathbb{Z}\left[z^{2}\right]$, and that each such polynomial occurs for some $L$, except for knots $(n=1)$ where we pose additionally the condition $[\nabla]_{0}=1$.

Since we have $2 \max \operatorname{deg} \Delta \leq 1-\chi$ for every link, it is clear that in any diagram $D$,

$$
2 \max \operatorname{deg} \Delta(D) \leq 1-\chi(D)
$$

The proofs of Theorem 2.2 of [50], [16] (for alternating diagrams $D$ ) and [13] (for positive diagrams $D$ ) follow from showing that (9) becomes an equality,

$$
2 \max \operatorname{deg} \Delta(D)=1-\chi(D) .
$$

Further, Murasugi proved a multiplicativity of the maximal (possible) coefficients under Murasugi sum.

TheOrem 2.6 (Murasugi [51]; see also [55]). If a diagram $D$ decomposes as Murasugi sum $A * B$, then

$$
[\Delta(D)]_{(1-\chi(D)) / 2}=[\Delta(A)]_{(1-\chi(A)) / 2} \cdot[\Delta(B)]_{(1-\chi(B)) / 2} \cdot
$$

Note also that

$$
\chi(A * B)=\chi(A)+\chi(B)-1 .
$$

The following property of the Alexander polynomial of fibered links is well known. We call a polynomial $\Delta$ in $\mathbb{Z}\left[t^{ \pm 1}\right]$ monic if max cf $\Delta= \pm 1$.

TheOREM 2.7 (See, e.g., [62]). If $L$ is fibered, then $2 \max \operatorname{deg} \Delta_{L}=1-\chi(L)$ and $\Delta_{L}$ is monic. 
2.5. The signature. The signature $\sigma$ is a $\mathbb{Z}$-valued invariant of knots and links, originally defined in terms of Seifert matrices [62]. We have that $\sigma(L)$ has the opposite parity to the number of components of a link $L$, whenever the determinant $\operatorname{det}(L)=\left|\Delta_{L}(-1)\right| \neq 0$. This in particular always happens for $L$ being a knot (since $\Delta_{L}(-1)$ is always odd in this case), so that $\sigma$ takes only even values on knots. Most of the early work on the signature was done by Murasugi [52], who showed several properties of this invariant.

Then, for links $L_{ \pm, 0}$ with diagrams as in (5), we have

$$
\begin{gathered}
\sigma\left(L_{+}\right)-\sigma\left(L_{-}\right) \in\{0,1,2\}, \\
\sigma\left(L_{ \pm}\right)-\sigma\left(L_{0}\right) \in\{-1,0,1\} .
\end{gathered}
$$

(Note: In the first property, one can also have $\{0,-1,-2\}$ instead of $\{0,1,2\}$, since other authors, like Murasugi, take $\sigma$ to be with opposite sign. Thus, (12) not only defines a property, but also specifies our sign convention for $\sigma$.)

The following further property is very useful: $\sigma(! L)=-\sigma(L)$, where $! L$ is the mirror image of $L$.

If $K$ is a positive knot, then $\sigma(K)>0$ [11]. One goal of [61] was to improve and extend this result. In particular, it was found that $\sigma(K) \geq 4$ if $g(K) \geq 2$; furthermore, $\sigma(K)>0$ if $K$ is almost positive or 2-almost positive, except a twist knot. See [70], [65], [73] for previously written (though not identical) proofs. We showed in [75] also that for $K$ positive, $\sigma(K) \geq 6$ if $g(K) \geq 4$ and $K \neq 14_{45657}$.

2.6. Gauß sum invariants. We recall briefly the definition of Gauß sum invariants. They were introduced first in [19] for braids, and later [20], [59] for knots. It is known that all they give formulas for Vassiliev invariants [7]. (See $§ 2,3$ of [59], for example, for more details.)

Definition 2.9 ([20]). A Gauß diagram of a knot diagram is an oriented circle with arrows connecting points on it mapped to a crossing and oriented from the preimage of the undercrossing (underpass) to the preimage of the overcrossing (overpass).

We will call the two arrow ends the hook and tail.

EXAmple 2.1. As an example, Figure 8 shows the knot $6_{2}$ in its commonly known (alternating) diagram and the corresponding Gauß diagram.

The simplest (non-trivial) Vassiliev invariant is the Casson invariant $v_{2}=$ $[\nabla]_{2}[5]$, with $-6 v_{2}=V^{\prime \prime}(1)$, for which Polyak-Viro [59], [60] gave the simple Gauß sum formula

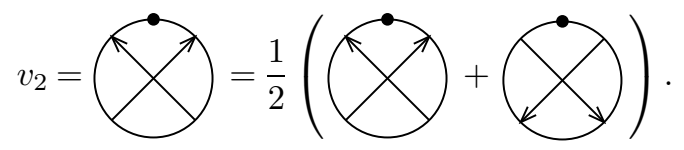



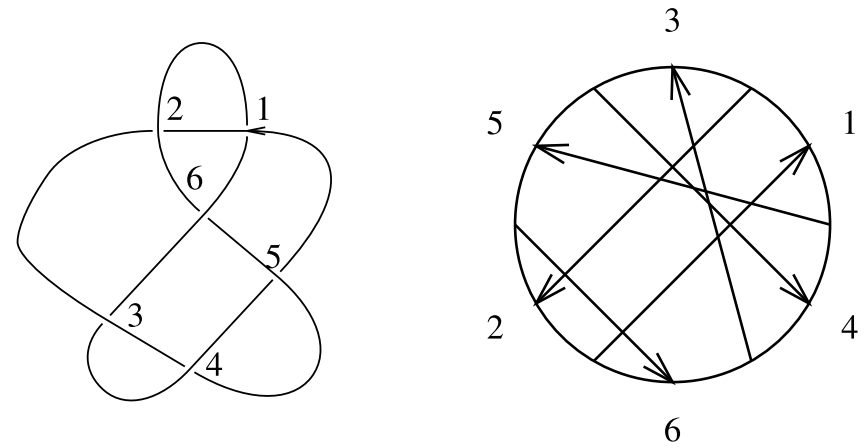

Figure 8 . The standard diagram of the knot $6_{2}$ and its Gauß diagram.

Here the point on the circle corresponds to a point on the knot diagram, to be placed arbitrarily except on a crossing. (The expression does not alter with the position of the basepoint; we will hence have, and need, the freedom to place it conveniently.)

A Gauß diagram $\Gamma$, occurring in a formula as (14) of an invariant, must be read as follows. It is understood as an integer-valued map on knot diagrams $D$. It evaluates by summations over all appropriate embeddings of $\Gamma$ into the Gauß diagram $G(D)$ of $D$. (That is, the arrows of $\Gamma$ should match a subset of arrows of $G(D)$.) For each embedding, the contribution summed, called weight, is made up of the writhes of the crossings in $D$ corresponding to the arrows of $G(D)$ matching $\Gamma$; by default, the weight is the product of these writhes.

Other formulas Polyak-Viro, and also Fiedler, gave for the degree-3Vassiliev invariant $v_{3}$. To make precise which variation of the degree-3Vassiliev invariant we mean, we have

$$
v_{3}=-\frac{1}{12} V^{\prime \prime}(1)-\frac{1}{36} V^{(3)}(1) .
$$

Fiedler's formula for $v_{3}[20]$, [21] reads ${ }^{2}$

$$
4 v_{3}=\sum_{(3,3)} w_{p} w_{q} w_{r}+\sum_{(4,2) 0} w_{p} w_{q} w_{r}+\frac{1}{2} \sum_{p, q \text { linked }}\left(w_{p}+w_{q}\right),
$$

where the configurations are

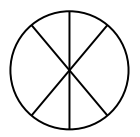

$(3,3)$

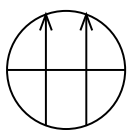

$(4,2) 0$

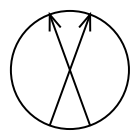

$p, q$ linked

${ }^{2}$ Note the factor 4 by which (15) differs from the definition in [68]. 
Here chords depict arrows which may point in both directions (i.e., a chord in $\Gamma$ matches an arrow in $G(D)$ of either orientation), and $w_{p}$ denotes the writhe of the crossing $p$.

If two chords $p$ and $q$ intersect, we call the corresponding crossings linked and write $p \cap q$. Otherwise, we say $p$ and $q$ are unlinked and write $p \not \subset q$. In that case, the crossing, whose over-pass (arrow head) is followed in counterclockwise orientation of the circle by the under-pass (arrow tail) of the other crossing is called distinguished. (In the third diagram of (16) it is the arrow going from lower right to upper left.)

A configuration will be denoted below as a pair $(p, q)$ or triple $(p, q, r)$ of crossings; hereby, no regard is given to their order, that is, which crossing matches which arrow in a Gauß diagram.

For a given Gauß diagram formula and fixed diagram to evaluate it on, we call a configuration positive, negative or neutral, depending on whether its weight is positive, negative, or zero, respectively. (In the formulas we use, we have no neutral three-arrow configurations.)

The below is an easy observation on Gauß diagrams, already known in [18], and named "even valence" in [68].

LEMMA 2.1 ("Even valence"). The number of crossings linked with a given one is always even.

There is a (similarly obvious) refinement of this condition in positive diagrams.

LEMMA 2.2 ("Extended even valence" [68]). In a positive diagram, each crossing is distinguished in exactly one half of the linked pairs it is contained in.

A further property of Gauß diagrams observed in [68] is the following.

LEMMA 2.3 ("Double connectivity" [68]). If $a \cap b, a \cap c$, but $b \nsupseteq c$, then there is a $d \neq a$ with $d \cap b$ and $d \cap c$.

We note, that $v_{3}$ is asymmetric, that is, $v_{3}(! K)=-v_{3}(K)$, while $v_{2}$ is symmetric, that is, $v_{2}(K)=v_{2}(! K)$. The latter property also justifies the second equality in (14). Moreover, $v_{2}$ and $v_{3}$ are integer-valued (even if for $v_{3}$ this is not quite obvious from its Gauß diagram formula).

2.7. Genus generators. Now let us recall, from [67], [73], some basic facts concerning knot generators of given genus. An explanation is given also in Section 5.3 in [14]. (There are several equivalent forms of these definitions, and we choose here one that closely leans on the previously set up terminology of Gauß diagrams.) We will also fix some notations and conventions used below.

Definition 2.10. Let $D$ be a knot diagram, and $p$ and $q$ be crossings.

(1) We call $p$ and $q$ equivalent, $q \simeq p$, if for all $r \neq p, q$ we have $r \cap p \Longleftrightarrow r \cap q$. 
(2) We call $p$ and $q \sim$-equivalent and write $p \sim q$ if $p$ and $q$ are equivalent and $p 內 q$.

(3) Similarly $p$ and $q$ are called $\approx$-equivalent, $p \approx q$, if $p$ and $q$ are equivalent and $p \cap q$.

(4) Finally, $p$ and $q$ are called twist equivalent, if there is a sequence of crossings $p=p_{0}, \ldots, p_{n}=q$ such that $p_{i}$ and $p_{i+1}$ form a clasp (cf. Definition 2.1).

It is an exercise to check that $\sim$ is an equivalence relation, and two crossings are $\sim$-equivalent if and only if after a sequence of flypes they can be made to

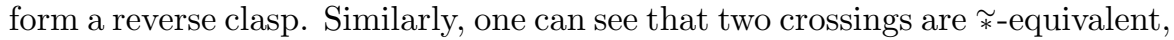
if and only if by flypes they can be joined into a parallel clasp.

Definition 2.11. Call two crossings $p$ and $q$ Seifert equivalent, $p \sim_{S} q$, if they connect the same two Seifert circles.

Let us record an easy but useful observation.

LEMma 2.4. Two $\approx$-equivalent crossings are Seifert equivalent. The converse is true in special diagrams.

A -equivalence class consisting of one crossing is called trivial, a class of more than one crossing non-trivial. We call a -equivalence class sometimes also a group of crossings.

Definition 2.12. Let $t(D)$ be the number of $\sim$-equivalence classes of $D$. For $i=1, \ldots, t(D)$ let $t_{i}(D)$ be the number of crossings in the $i$ th $\sim$ equivalence class. Then for $i=1, \ldots, t(D)$ and $j=1, \ldots, t_{i}(D)$ let $p(D, i, j)$ be the $j$ th crossing in the $i$ th $\sim$-equivalence class of $D$.

Definition 2.13. A $\bar{t}_{2}^{\prime}$ move or twist at a crossing $x$ in a diagram $D$ is a move, which creates a pair of $\sim$-equivalent crossings to $x$ of the same sign as $x$. (This is well-defined up to flypes.) Accordingly, we can distinguish between a positive and a negative twist (we will mostly use the former).

DEFinition 2.14. An alternating diagram $D$ is called $\bar{t}_{2}^{\prime}$ irreducible or a generating diagram, if $t_{i}(D) \leq 2$ for $i=1, \ldots, t(D)$. An alternating knot $K$ is called generator if some of its alternating diagrams are generating. The diagrams obtained from $D$ by $\bar{t}_{2}^{\prime}$ moves and crossing changes form the sequence or series $\langle D\rangle$ of $D$.

Observe that, since $\sim$-equivalence is invariant under flypes, Theorem 2.3 implies that some alternating diagram of $K$ is generating if and only if all its alternating diagrams are so.

A flype in Figure 4 is called trivial if one of the tangles contains only crossings equivalent to the crossing admitting the flype. 


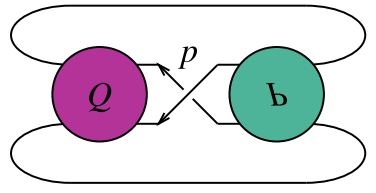

type A

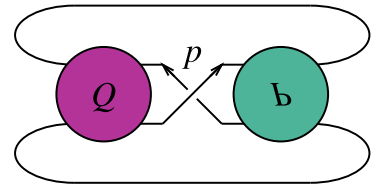

type B

Figure 9. A flype of types A and B.

We introduced (see [76]) a distinction of flypes according to the orientation near the crossing $p$ at which the flype is performed. See Figure 9, and compare with the right of Figure 4 (again $p$ is meant up to reflection).

A flype is of type $A$ if the strand orientation is so that strands on the left/right side of each tangle are directed equally with respect to the tangle (i.e., both enter or both exit). Otherwise it is a flype of type $B$. So the property to admit a type $A$ resp. type $B$ flype is an invariant of the $\approx$ resp. $\sim$-equivalence class.

An important observation is that each crossing admits at most one of the types $\mathrm{A}$ and $\mathrm{B}$ of flypes, and this remains so after applying any sequence on flypes on the diagram.

THEOREM 2.8 ([9], [67]). There exist only finitely many generators of given genus. All diagrams of that genus can be obtained from diagrams of these generators, under $\bar{t}_{2}^{\prime}$ twists, flypes, and crossing changes.

In [74], we obtained rather sharp estimates on the maximal number of crossings and $\sim$-equivalence classes of generators. This, together with the examples in [76], determined the maximal crossing number of a knot generator to be $10 g-7$ for genus $g \geq 2$. In practice (in particular as we will see below), it is important to obtain the list of generators for small genus. Genus one is easy, and also observed independently.

ThEOREM 2.9 ([67]; see also [9], [63]). There are two generators of genus one, the trefoil and figure-8-knot.

Genus two and three require much more work. For suggestive reasons, it is sufficient to find prime diagrams, and by Theorem 2.1, prime generators.

THEOREM 2.10 ([73]). There are 24 prime generators of genus two. They have at most $9 \sim$-equivalence classes. There are 4017 prime generators of genus 3 , of at most $15 \sim$-equivalence classes.

A classification, by means of obtaining the list of prime generators, is also possible for genus $g=4$, and is explained in [75]. Note that, with the rapid 
growth of complexity, apart from a computational challenge, each new generator list required an entirely different (and considerably more efficient) method to compile.

It follows from Theorem 2.3 that the series of different (alternating) diagrams of the same generating knot are equivalent up to mutations. For tests based on the Jones polynomial, which is invariant under mutations, it is legitimate that a priori we fix throughout Section 3 and Section 4 a single specific diagram $D$ for each generator $K$, and work only with (the series of) this diagram. Then we write $t(K)=t(D), t_{i}(K)=t_{i}(D)$ and $p(K, i, j)=p(D, i, j)$, and speak of the sequence or series $\langle K\rangle=\langle D\rangle$ of $K$.

For the purpose of Section 3 and Section 4, let us parametrize diagrams in the series of a generator $K$ as $K\left(x_{1}, \ldots, x_{l}\right)$, where $l=t(K)$, the $l \sim$ equivalence classes of $K$ are ordered in some fixed way, and $x_{i}$ are defined as follows.

For a trivial $\sim$-equivalence (i.e., $t_{i}=1$ ), $x_{i} \geq 1$ means a $\sim$-equivalence class of $2 x_{i}-1$ positive crossings, or alternatively, the result of applying $\left(x_{i}-1\right)$ $\bar{t}_{2}^{\prime}$-moves to a single positive crossing in the generator. If $x_{i} \leq 0$, then we have a $\sim$-equivalence class of $1-2 x_{i}$ negative crossings, that is, a negative crossing with $\left(-x_{i}\right) \bar{t}_{2}^{\prime}$-moves applied.

For a $\sim$-equivalence class of $t_{i}=2$ crossings, $x_{i}>0$ means a $\sim$-equivalence class of $2 x_{i}$ positive crossings, or alternatively, the result of applying $\left(x_{i}-1\right)$ $\bar{t}_{2}^{\prime}$-moves to one of the two positive crossings in the generator. The parameter $x_{i}=0$ designates that the two crossings have opposite sign, that is, form a trivial clasp after flypes. Finally, $x_{i}<0$ indicates $-2 x_{i}$ negative crossings in the $\sim$-equivalence class, that is, the result of $\left(-1-x_{i}\right) \bar{t}_{2}^{\prime}$-moves on one of the negative two crossings in the generator.

This convention will remain valid for the rest of the paper. Note that it implies that we discard diagrams with crossings of different sign within the same $\sim$-equivalence class (unless $x_{i}=0$ and $t_{i}=2$ ). Such diagrams have a trivial clasp after flypes, and are usually of little interest.

\section{Genus-minimizing almost alternating diagrams}

We start with the results and examples concerning almost alternating diagrams, since they are easier to obtain and argue about.

We will need the following lemma that easily combines some less easy ingredients.

LEMMA 3.1. An almost positive diagram is genus-minimizing if and only if the negative crossing has no Seifert equivalent one (see Definition 2.11).

Proof. An almost positive diagram decomposes under Murasugi sum into special alternating blocks and one almost special alternating one $D_{0}$. By Theorem 2.5, we need to look only at $D_{0}$. The claim for $D_{0}$ is an easy consequence of [71, Corollary 5] (see also the proof of Theorem 5 of [71]). 


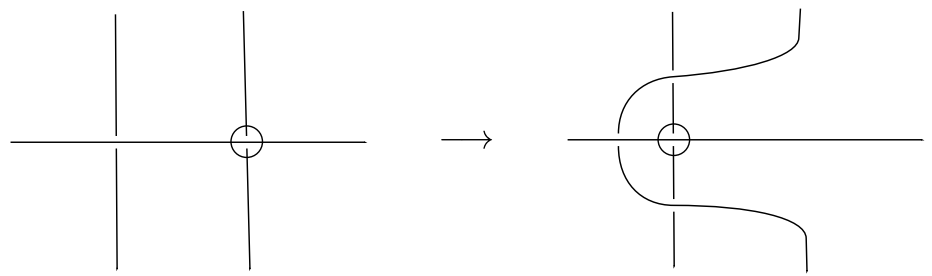

Figure 10. A tongue move.

This corollary states that if in an almost special alternating diagram $D_{0}$ the negative crossing $p$ has no Seifert equivalent (positive) one, then $D_{0}$ is of minimal genus and satisfies (10). Notice that the property easily extends to an equivalence: if $p$ has a Seifert equivalent crossing $q$, by a flype (in a special diagram), one can include $p$ in a trivial parallel clasp with $q$ (see Lemma 2.4). Then $D_{0}$ is not of minimal genus (and in particular (10) fails for $D_{0}$ ).

Proof of Theorem 1.1. Adams introduced a tongue move allowing to build more complicated almost alternating diagrams from a given one. This move is shown in Figure 10. Herein the crossing needed to be switched to obtain an alternating diagram is encircled; we call this crossing the dealternator.

Now let us trace the effect of a tongue move on the Seifert circle picture. The result depends somewhat on the orientation of the strands. Let $c$ be the overpassing strand of the dealternator $p$ and $a$ the underpassing strand. Let $b$ be the underpassing strand of one of the crossings $q$ neighbored to $p$ along $c$. We can assume that $q \neq p$ since otherwise $p$ is nugatory.
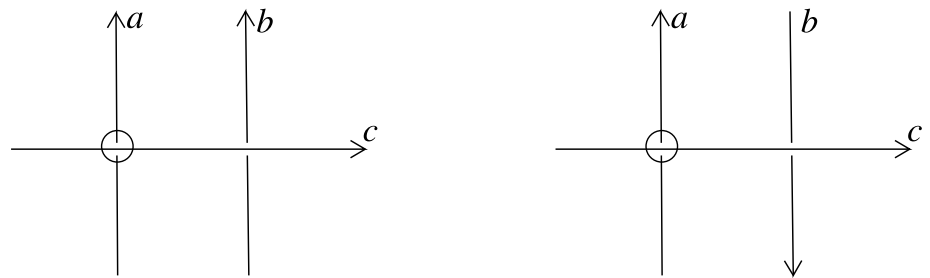

If now $a$ and $b$ point in the same direction with regard to $c$, then it is easy to see that a tongue move of $a$ and $b$ does not alter the Seifert circle picture, while it creates two new crossings. Then the diagram genus goes up by one. If $a$ and $b$ are oppositely oriented with respect to $c$, then the number of Seifert circles is altered at most by \pm 2 . Thus the diagram genus may or may not increase, but it certainly does not decrease.

Since the orientation of $c$ does not matter in this argument, there are two strands on which a tongue move with strand $a$ can be applied, the ones that cross $c$ directly before and after $a$. Call them $b_{1}$ and $b_{2}$. If one or both of 
(i)

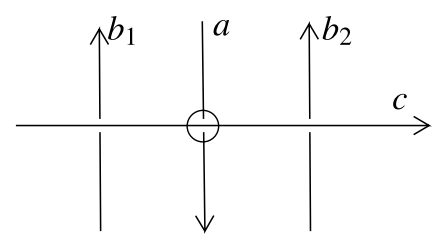

(ii)

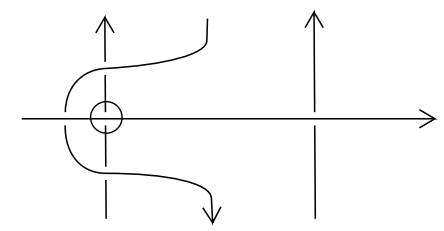

(iii)

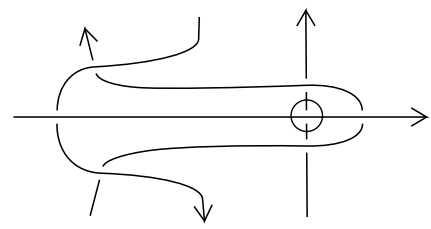

Figure 11. Augmenting diagram genus using tongue moves.

TABle 1. Properties of the $K T$ and $C$ knots

\begin{tabular}{lcccc}
\hline Name & Perko number & KnotScape number & Sliceness & Genus \\
\hline$K T$ & 473 & 409 & Yes & 2 \\
$C$ & 471 & 401 & Unknown & 3 \\
\hline
\end{tabular}

them cross $c$ in the same direction as $a$, this move would augment the diagram genus. Otherwise $b_{1,2}$ have equal orientation with respect to $c$. Apply first a tongue move with $a$ and $b_{1}$, and now that $b_{1,2}$ intersect $c$ consecutively, a tongue move with $b_{1,2}$ (see Figure 11). At least the second of those moves strictly augments the genus.

The claim follows by iterating this procedure.

This simple argument shows that we can certainly not hope every almost alternating diagram to have minimal genus. More interestingly, however, we will show that for some knots no minimal genus diagram exists at all.

We recall the two $\Delta=1$ knots of 11 crossings, the Kinoshita-Terasaka $K T$ and Conway $C$ knots. Table 1 should help distinguishing them. (The Perko number refers to [58]; the genera have been determined by Gabai [26].) See Figure 12 for diagrams of these knots. 

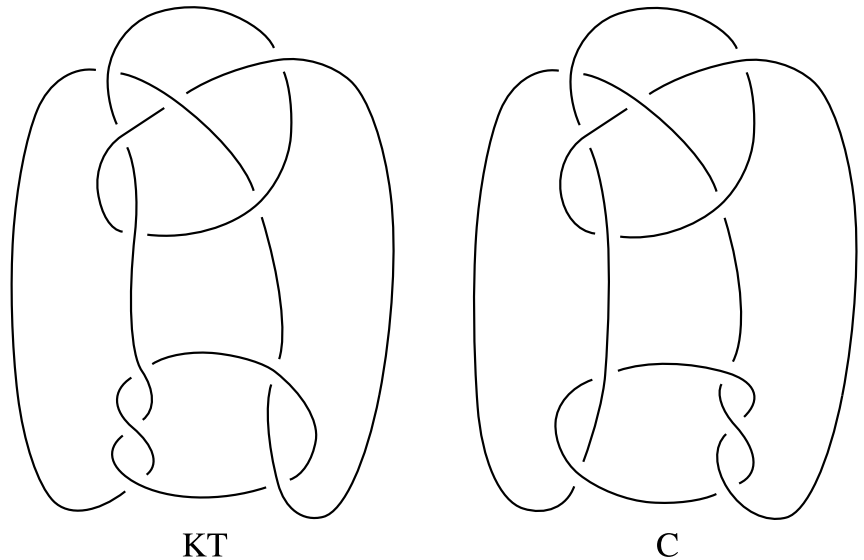

FiguRE 12. Diagrams of the Kinoshita-Terasaka and Conway knots.

Proof of Theorem 1.2. Part (a). We use an observation from [71]. The Kinoshita-Terasaka knot has genus two [26, Figure 5], and is almost alternating by the verification in [3], [4], while the calculation in [43, Example 11.1] gives $\max \operatorname{deg}_{m} P=6$, so that by Morton's inequality [49],

$$
\max \operatorname{deg}_{m} P(K) \leq 2 \tilde{g}(K),
$$

we have $\tilde{g}=3$. (A genus three canonical surface is not too hard to find, see below.) This knot thus does not have any diagram whatsoever of minimal genus.

Now $K T$ unknots in its diagram in Figure 12 by one crossing change in the upper tangle of 6 crossings. (We let the reader find it.) Call this crossing $p$. Applying $\bar{t}_{2}^{\prime}$-twists at this crossing (without switching), we obtain a series of diagrams $D_{i}$ of knots $K_{i}$. We choose the indexing so that $K_{0}$ is the unknot, $K_{1}=K T$, and $D_{i}$ is obtained from $D_{1}$ by $(i-1) \bar{t}_{2}^{\prime}$-twists at $p$, henceforth assuming $i>0$. Since $\Delta\left(K_{0}\right)=\Delta\left(K_{1}\right)=1$, the skein relation for $\Delta$ immediately implies that $\Delta\left(K_{i}\right)=1$ for all $i$. Thus, $K_{i}$ are trivial or non-alternating [50]. Now consider the skein polynomial. Since $p$ is negative, its skein relation implies

$$
\left(-l^{2}+1\right) P\left(! K_{i-1}\right)+l^{2} P\left(! K_{i-2}\right)=P\left(! K_{i}\right) .
$$

Since $P\left(K_{0}\right)=1$ and $\max \operatorname{deg}_{m} P\left(K_{1}\right)=6$, we easily find $\max \operatorname{deg}_{m} P\left(K_{i}\right)=6$ and for $i>1$

$$
\max \operatorname{deg}_{l}\left[P\left(! K_{i}\right)\right]_{m^{6}}=\max \operatorname{deg}_{l}\left[P\left(! K_{i-1}\right)\right]_{m^{6}}+2 .
$$

Thus by (17) the knots have $\tilde{g}\left(K_{i}\right) \geq 3$, and are distinct. Now $g\left(K_{0}\right)=0$ and $g\left(K_{1}\right)=2$, so that by Corollary 2.4 of [29] (as explained in Remark 11.1 of 

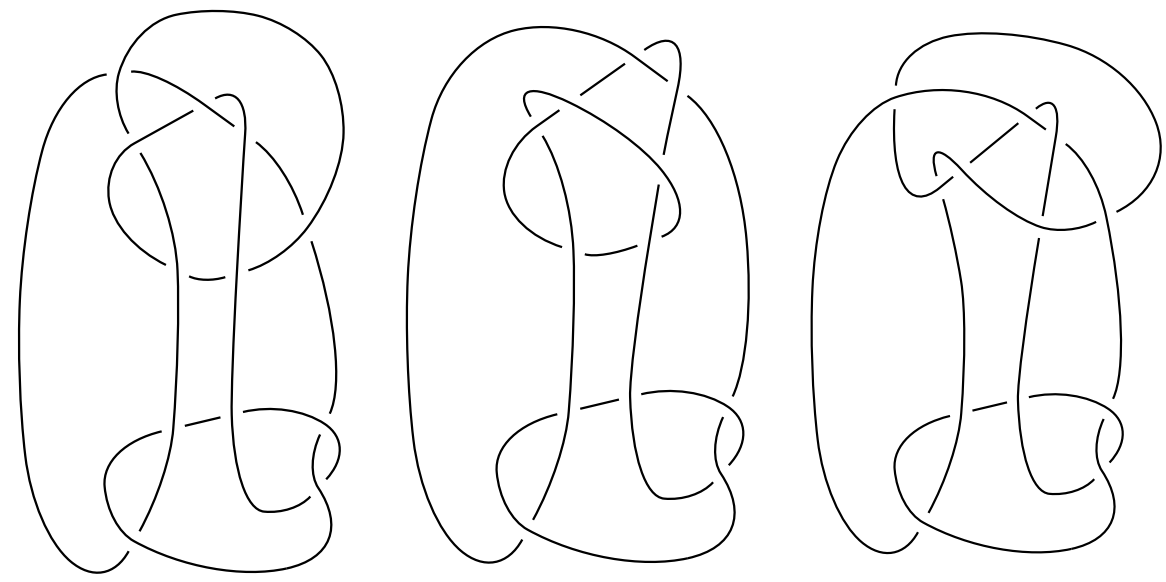

Figure 13. Moves of the Conway knot diagram in Figure 12 to one of minimal genus.

[73]) we have $g\left(K_{i}\right)=2$ for all $i>0$. In particular, the $K_{i}$ are non-trivial, and so non-alternating, and $\tilde{g}\left(K_{i}\right)>g\left(K_{i}\right)$. That they are almost alternating follows in the same way as for $K T$ in the verification in [3], [4], since the twists at $p$ keep the upper tangle of $D_{i}$ alternating, and do not affect the wrongly mirrored $(3,2)$-pretzel tangle in the bottom.

Part (b). Now consider the other knot $C$. This knot has genus 3 , and has a diagram of minimal genus. Gabai gives its canonical surface, slightly isotoped, in his op. cit. drawing. (A mutation of this diagram gives a canonical surface of genus 3 for $K T$.) But we need a particular sequence of moves that turns the diagram in Figure 12 into a genus 3 diagram. See Figure 13. Again the diagram of $C$ in Figure 12 has a negative unknotting crossing $p$ in the upper tangle. The sequence of moves in Figure 13 is chosen so that $p$ is preserved, and switching $p$ or applying $\bar{t}_{2}^{\prime}$ moves at $p$ commutes with these moves. Now consider $K_{i}$ defined an analogy to $K T$. Most of the argument repeats, except that now the moves in Figure 13 show $\tilde{g}\left(K_{i}\right) \leq 3$, and Gabai's result that $g\left(K_{i}\right)=3$, so that $\tilde{g}\left(K_{i}\right)=g\left(K_{i}\right)=3$, as we claimed. The diagrams obtained after $\bar{t}_{2}^{\prime}$ moves at $p$ from Figure 12 are still needed to ensure that $K_{i}$ are almost alternating. Now assume that some $K_{i}$ had an almost alternating diagram $D$ of minimal genus. Then from [71] we would have that

$$
\max \operatorname{deg} \Delta\left(K_{i}\right)=g(D)=g\left(K_{i}\right)
$$

(see below), but max deg $\Delta\left(K_{i}\right)=0$, a contradiction.

Thus for the rest of the proof of this part, we explain (19). The canonical surface of $D$ is the Murasugi sum of the canonical surfaces of its blocks (see Definition 2.5). Then $D$ is a Murasugi sum of an almost special alternating 
block with special alternating blocks. The latter are of minimal genus and satisfy (10). The property (10) for genus-minimizing almost special alternating diagrams $D$ is an easy consequence of the proof of Lemma 3.1. By Murasugi [51], and also Murasugi-Przytycki [55], the top coefficient of the Alexander polynomial is multiplicative under Murasugi sum (Theorem 2.6). Murasugi sum also preserves the minimal genus property of the canonical surface by the work of Gabai (Theorem 2.5). This completes the explanation of (19).

Part (c). Of course, if a knot is supposed to have a diagram of minimal genus and $\max \operatorname{deg} \Delta=g$, both Morton's inequality and the condition of [71] on the Alexander polynomial do not apply, and we must seek a different method. The one we use was explained in $[73, \S 9]$ and involves values of the Jones polynomial at roots of unity.

Consider an almost alternating genus two diagram $D$, which does not simplify to an alternating one. If $D$ represents a prime non-alternating knot, it is easy to see that $D$ is prime. Thus from now on consider only prime generators $K$. We write $l=t(K)$ for the number of $\sim$-equivalence classes.

It is clear that the dealternator of $D$ must be single in its -equivalence class. Formally written, $D=K\left(x_{1}, \ldots, x_{l}\right)$, where $K\left(\tilde{x}_{1}, \ldots, \tilde{x}_{l}\right)$ is the alternating generator diagram, and (up to taking the mirror image of $D$ ) with $g_{i}=1-t_{i} / 2$, we have $h_{i}=\left(x_{i}-g_{i}\right)\left(\tilde{x}_{i}-g_{i}\right)>0$ except for exactly one $i=1, \ldots, l$, for which $h_{i}=-1 / 4$.

Now consider for $n \in \mathbb{N}$ the residue

$$
V_{n}(D)=V(D) \bmod \frac{t^{2 n}-1}{t^{2}-1} .
$$

It was observed (by work of Przytycki) in Theorem 9.2 and Corollary 9.1 of [73] that for $D=K\left(x_{1}, \ldots, x_{l}\right)$, the value $V_{n}(D)$ depends only on $x_{i} \bmod n$. (Note that $K\left(x_{1}, \ldots, x_{i}+n, \ldots, x_{l}\right)$ differs from $K\left(x_{1}, \ldots, x_{i}, \ldots, x_{l}\right)$ by a $\bar{t}_{2 n}$ move.) Now one can adjust the sign of $x_{i}$ by adding a multiple of $n$. Thus if $\hat{K}$ is prime and non-alternating, and has an almost alternating diagram of genus 2, then, depending on the sign of the dealternator, one of $V_{n}(\hat{K})$ or $V_{n}(! \hat{K})$ must be element in the set

$$
\mathcal{V}_{n}:=\left\{\begin{array}{c}
D=K\left(x_{1}, \ldots, x_{l}\right), K \text { prime generator of } \\
V_{n}(D): \text { genus } 2, \text { all } x_{i}>0 \text { except exactly one } \\
i=1, \ldots, l, \text { where } t_{i}=1 \text { and } x_{i}=0
\end{array}\right\} .
$$

To find knots, where this is not the case, the proper value of $n$ to take depends on a balance between the strength of the invariant $V_{n}$ and the number of invariants to compute. Since $V_{n}$ depends only on $x_{i} \bmod n$, for the calculation of $\mathcal{V}_{n}$ at most $n^{l}$ polynomials in the sequence of $K$ are needed. For genus 2, we have 24 prime generators $K$, with $l=t(K) \leq 9$. In [73, §9], we found that $n=5$ is a good choice. Then $\mathcal{V}_{5}$ can be calculated similarly as there, and is found to have 86,701 elements. For the computations in [73], we also compiled 
prime $\leq 16$ crossing non-alternating knots with $\max _{\operatorname{meg}} P \leq 4$. A check in this list shows that the pretzel knots $(-3,-3,3,3,3) 1_{184486},(-3,3,-3,3,3)$ $15_{184487}$, and $(3,3,3,3,-3) 15_{197572}$ (see also Figure 10 in [73]) are the desired examples $\hat{K}$. For all of them neither $V_{5}(\hat{K})$ nor $V_{5}(! \hat{K})$ belong to $\mathcal{V}_{5}$. (Note that the first two have the same polynomial, being mutants.) Since $\max \operatorname{deg} \Delta=2$, and the pretzel diagrams have genus 2 , the knots have diagrams of minimal genus. Also $[\Delta(t)]_{2}=-14$ resp. 4 , so that the knots are obviously not fibered. It remains to argue why they are almost alternating. It is easy to see that they are not alternating (for example, their Jones polynomials violate the properties of [78] to have alternating coefficients and to be monic on either side). Then it is an easy exercise using Conway [12] transformations that any non-alternating pretzel, in fact, more generally Montesinos, link is almost alternating. (See Proposition 6.5 in [1] or [3, §3] - for $15_{197572}$ Theorem 3.1(i) there even applies directly, or the remarks in $\S 2$ of [2].)

To obtain infinitely many examples, remember that $V_{5}$ is invariant under five $\bar{t}_{2}^{\prime}$ twists (applied at the same crossing). Thus, the $\left(x_{1}, \ldots, x_{5}\right)$-pretzel knots with all $x_{i} \equiv \pm 3 \bmod 10$ have the same $V_{5}$. (We can choose the ' \pm ' for each $x_{i}$ independently, except that at least one should be positive and at least one negative.) The knots are prime for example by the criterion in [41]. If $x_{i} \equiv \pm 3 \bmod 20$, then $\Delta \bmod 2$ is preserved, and $[\Delta(t)]_{2} \neq \pm 1$. Now $[\Delta(t)]_{2}$ is easily seen to depend polynomially on the $x_{i}$, and this polynomial does not vanish for some particular values of $x_{i}$ (that satisfy these congruences). Then it does not vanish for generic such values, and for proper values (with signs that can be given a priori) becomes arbitrarily large, so that infinitely many of the knots are distinct. The knots are not alternating for $\sum \operatorname{sgn}\left(x_{i}\right)= \pm 1$, since by [44, example, p. 529] they have adequate non-alternating diagrams, and by [79] such prime knots are non-alternating. Then they are almost alternating by the above remark.

Part (d). Consider the $(2 a, 2 b, 2 c)$-pretzel diagram of a 3-component link, oriented so that it is special (all twists are reverse). The triples $(a, b, c)$ for which its canonical surface is a fiber were classified by Kanenobu [38] and Gabai [28]. (See in Gabai's paper Theorem 6.7, case 1(B) and (C), p. 538, and historical remark, p. 542.) For such triples, at least two of $a, b, c$ are \pm 1 . They are much fewer than those, for which $[\Delta]_{t}=a b+a c+b c= \pm 1$. The simplest example is $(-1,2,3)$. One easily finds in fact infinitely many $(2 a, 2 b, 2 c)$-pretzel diagrams with $[\Delta]_{t}= \pm 1$, whose canonical surfaces are not fibers. The previous triple extends for $k \in \mathbb{Z}$ to series like

$$
(a, b, c)=\left(-1+125 k^{2}+5 k, 2-25 k, 3+25 k\right) .
$$

Using plumbings of positive Hopf bands (of 1 full twist), in the way shown by example in Figure 11 of [71], one constructs from these pretzel diagrams genus two knots with $[\Delta]_{t^{2}}= \pm 1$. By [25], [27] a Hopf plumbing does not alter the fiber property. The knots obtained after plumbings from such surfaces 
are therefore not fibered. They are Montesinos, and hence alternating or almost alternating. But if they had an almost alternating diagram of minimal genus, then maxcf $\Delta= \pm 1$ implies by [71, Theorem 7] (modified as in the proof of Corollary 1.1) that they are fibered, a contradiction. The same contradiction arises from [51], assuming they were alternating. It remains to see that infinitely many of them are distinct. This can be done using $v_{2}$. One can find, for example, from (14), that, applying the plumbings as in Figure 11 of [71], it is an affine-linear combination of $a, b$ and $c$. Also, taking positive Hopf bands for plumbing ensures that all coefficients of this linear function (except possibly the absolute one) are non-zero.

Proof of Theorem 1.3. Recapture the final arguments in the previous proof. Now use for $(a, b, c)$ the values $(-1,1, n / 2), n \in 2 \mathbb{Z}$. Then by Kanenobu-Gabai, the $(2 a, 2 b, 2 c)$-pretzel surface is a fiber. Plumb Hopf bands of different sign near the \pm 2 half-twists to obtain the Montesinos knot with Conway [12] notation $(n, 22,-2-2)$. Since smoothing out a crossing in the group of $n$ gives the 2-component unlink, the Alexander polynomials of these knots are all the same. That the knots are distinct follows from studying $v_{3}$. It behaves polynomially in $n$ (see [66]), and a direct calculation shows that for $n=0$ we have $4_{1} \# 4_{1}$ with $v_{3}=0$, while for $n= \pm 2$ we obtain $10_{137}$ and its mirror image with $v_{3}= \pm 2$. The knots are of Montesinos type, and so almost alternating or alternating. The latter situation can occur only for finitely many. This follows from several results, for example, of [17] (by means of a bound on the crossing number of an alternating knot of given $\Delta(-1)$ ), or [13, Corollary 5.1] (by means of such a bound depending on max $\operatorname{deg} \Delta$ for $\max \operatorname{cf} \Delta= \pm 1)$.

REMARK 3.1. Since the fiber of $4_{1} \# 4_{1}$ is clearly a Hopf plumbing, the Melvin-Morton condition [45] on $\Delta$, used in [30] for $9_{42}, 9_{44}$ and $9_{45}$, does not apply to any of our last examples.

Proof of Corollary 1.1. This is a combination of Theorem 1.3 with the fact that there are only finitely many almost alternating knot diagrams whose canonical surfaces are fibers of given genus. This latter property is an easy consequence of [71, Theorem 7], and we explain how.

First, Theorem 7 in [71] is stated for almost positive diagrams. As noticed also there, though, it holds as well for almost alternating diagrams, since almost positive diagrams and almost alternating diagrams differ only by mirroring some blocks (in Definition 2.4). Mirroring preserves the fibering property, and by Gabai (Theorem 2.5), so does Murasugi sum. This explains, for almost alternating diagrams, the equivalence of conditions (2) and (4) in Theorem 7 in [71]. (Condition (1) also follows easily, and for (3) one can use Theorem 2.6, although those parts will not be needed here.) 
Then, for the finiteness we asserted, note first that because of (11) and $\chi\left(D_{i}\right) \leq 0$ for the blocks $D_{i}$ of $D$, the number of these blocks is at most $1-\chi(D)$. It is thus enough to see the finiteness for special diagrams $D$ with fixed $\chi(D)$ (since there is a finite number of ways to perform Murasugi sum). Now use the characterization in part (4) of the theorem. Observe that for a diagram $D$ as in Figure 8 in [71], the number of vertices of the graph on the left is at least 2 and equal to $2-\chi(D)$.

\section{Almost positive knots}

4.1. The degree of the Alexander polynomial. We will first show that the property 2 max deg $\Delta(L)=1-\chi(L)$ [16], [50], [13] extends to almost positive non-split links $L$, as stated in Theorem 1.5. Thus, we have for such $L$

$$
2 \max \operatorname{deg} \Delta(L)=1-\chi(L)=\max _{\operatorname{deg}_{m}} P(L) .
$$

Note that the second equality in (21) is from [71, Theorem 6], and much easier to prove than Theorem 1.5, although much less natural to interpret. The equality $\max _{\operatorname{leg}} P(L)=1-\tilde{\chi}(L)$ (that is, the exactness of one of the inequalities of [49]) would be its natural counterpart. This leads to the following question.

Question 4.1. Is $\chi(L)=\tilde{\chi}(L)$ for every almost positive link $L$ ?

An answer is difficult to give, despite that the examples below suggest as more likely a negative one.

For the proof of Theorem 1.5, we need to recall some terminology and results of [71], Definition 2.11, and Lemma 3.1.

Proof of Theorem 1.5. Consider an almost positive diagram $D$ of $L$. If $D$ is genus-minimizing, then we know the assertion from [71, Corollary 5]. We thus assume for the entire proof that $D$ is not genus-minimizing, that is, $\chi(D)=\chi(L)-2$. (Note that always $\chi(D) \geq \chi(L)-2$, as stated in (1).)

Let $p$ be the negative crossing of $D$. Let $a$ and $b$ be the Seifert circles connected by $p$, such that the overpass of $p$ moves from $b$ to $a$.

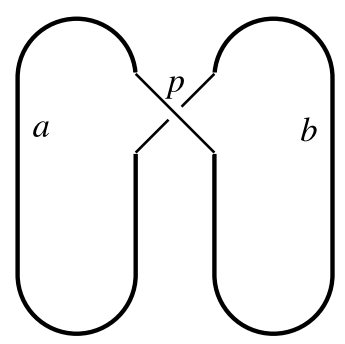


Let $q$ be the first positive crossing between $a$ and $b$ along $a$, when starting at $p$. (We know from Lemma 3.1 that such a crossing exists.)

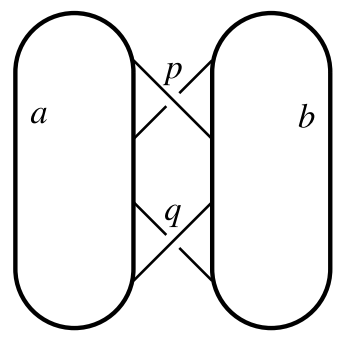

Label the regions into which $a, b, p$ and $q$ separate the plane $A, B, C$ and $E$ as follows.

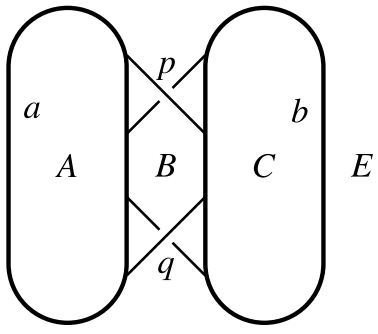

Note that by choice of $q$ there is no crossing in $D$ joining $a$ and $b$ within region $E$.

Let $e$ be the part of $a$ between $p$ and $q$ (that is, starting from $p$ and ending on $q$, in the direction of the orientation of $a$ ). Similarly, let $f$ be the part of $b$ between $q$ and $p$, and let $e^{\prime}$ and $f^{\prime}$ be the remaining parts of $a$ and $b$, resp.

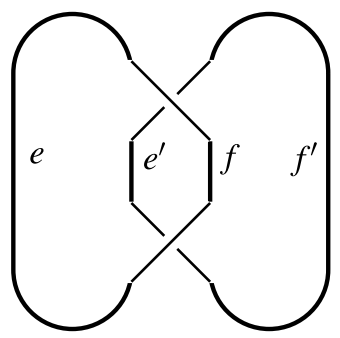

Let $e_{1}, \ldots, e_{n}$ be the Seifert circles in $E$ connected by a crossing to $e$, and $p_{1}, \ldots, p_{\nu}$ be those crossings. (Note that $\nu \geq n$, since there may be several crossings for one Seifert circle.) Let $e_{1}^{\prime}, \ldots, e_{n^{\prime}}^{\prime}$ be those Seifert circles in $A$ connected by a crossing to $e$, and $p_{1}^{\prime}, \ldots, p_{\nu^{\prime}}^{\prime}$ be the connecting crossings. Let $f_{1}, \ldots, f_{m}$ be the Seifert circles in $C$ connected by crossings $q_{j}$ to $f$, and $f_{1}^{\prime}, \ldots, f_{m^{\prime}}^{\prime}$ those in $B$ connected to $f$ by crossings $q_{j}^{\prime}$. See Figure 15 . 
Now consider the (binary rooted) skein tree of [13]. We describe how it is obtained, with some more details and modifications.

The root is labelled by $D$. In $D$ we distinguish a bridge $\beta$ (line segment consisting only of overcrossings), which starts before the overcrossing of $p$, and is extended in the orientation of the component maximally, that is, until an undercrossing occurs. Then each binary node of the tree looks like
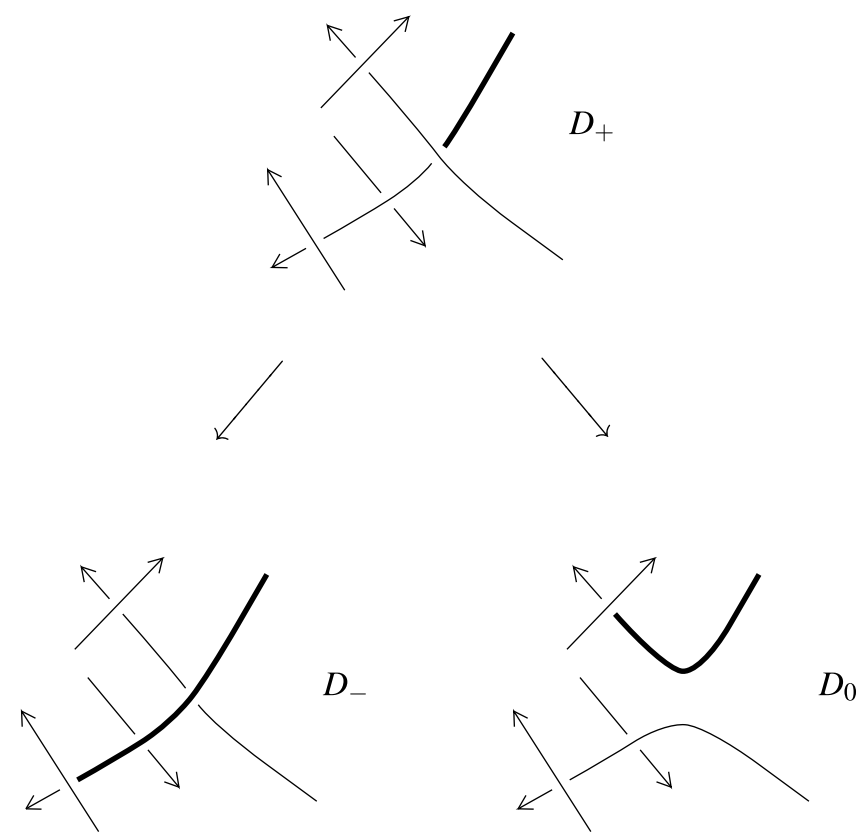

In other words, $\beta$ is always extended until the first undercrossing whose overcrossing does not belong to $\beta$. We do extend the bridge through undercrossings with itself.

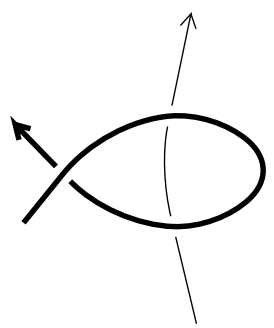

Then to build the two nodes below $D_{+}$switch resp. smooth out the undercrossing terminating the bridge $\beta$.

A terminal node is the one in which the bridge $\beta$ closes to a loop, that is, reaches its start. (This is not exactly what is a terminal node in the usual 


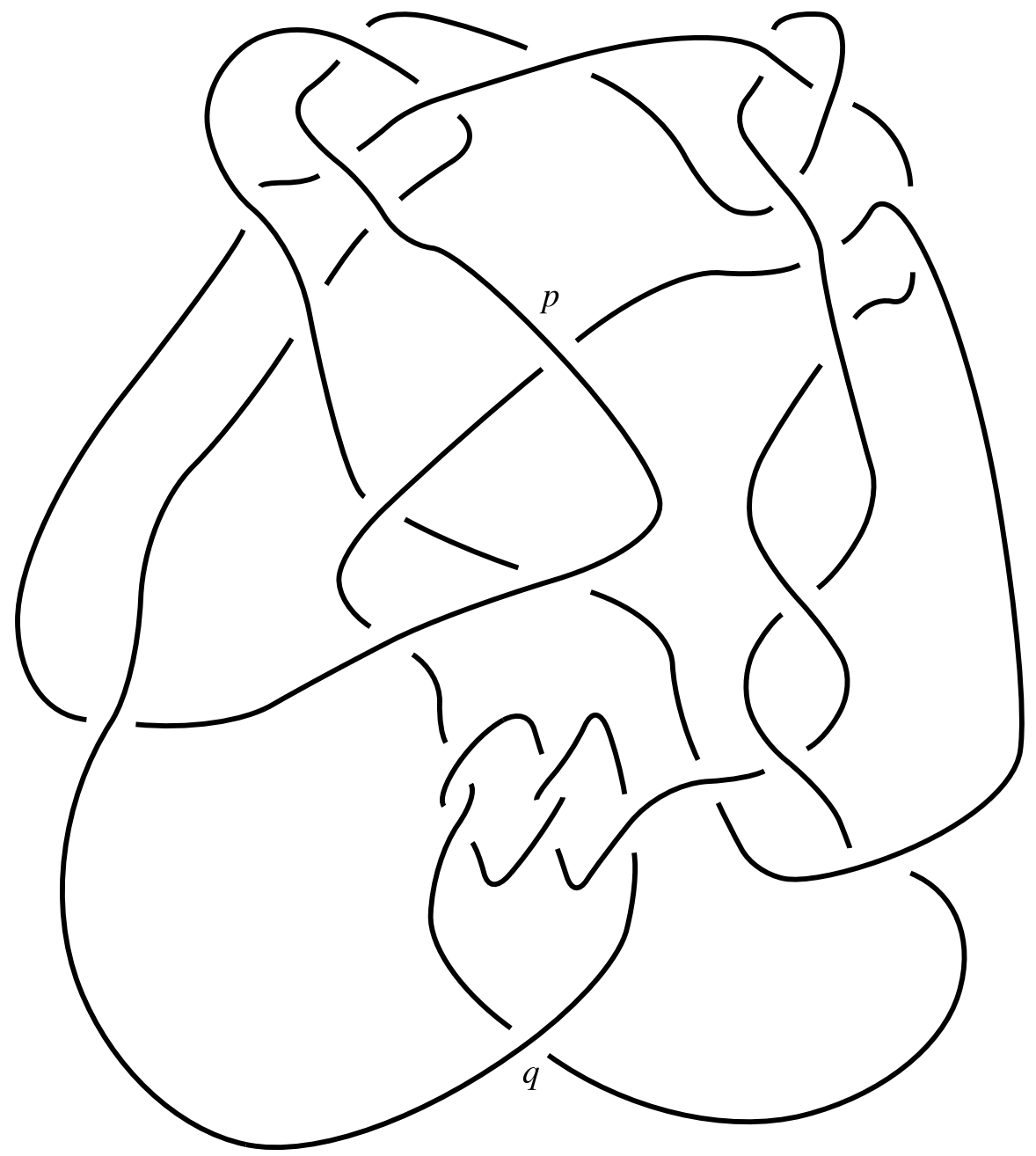

Figure 14. An almost positive link diagram, used to illustrate the proof of Theorem 1.5.

sense, described in [13], but it is sufficient and more suitable for our purpose here.) Note that in a terminal node the component marked by $\beta$ is trivial (i.e., unknotted, since any crossing with itself is passed by $\beta$ first as an overpass) and split from other components of the link (if there are any; since all crossings with such components are passed as an overpass). 


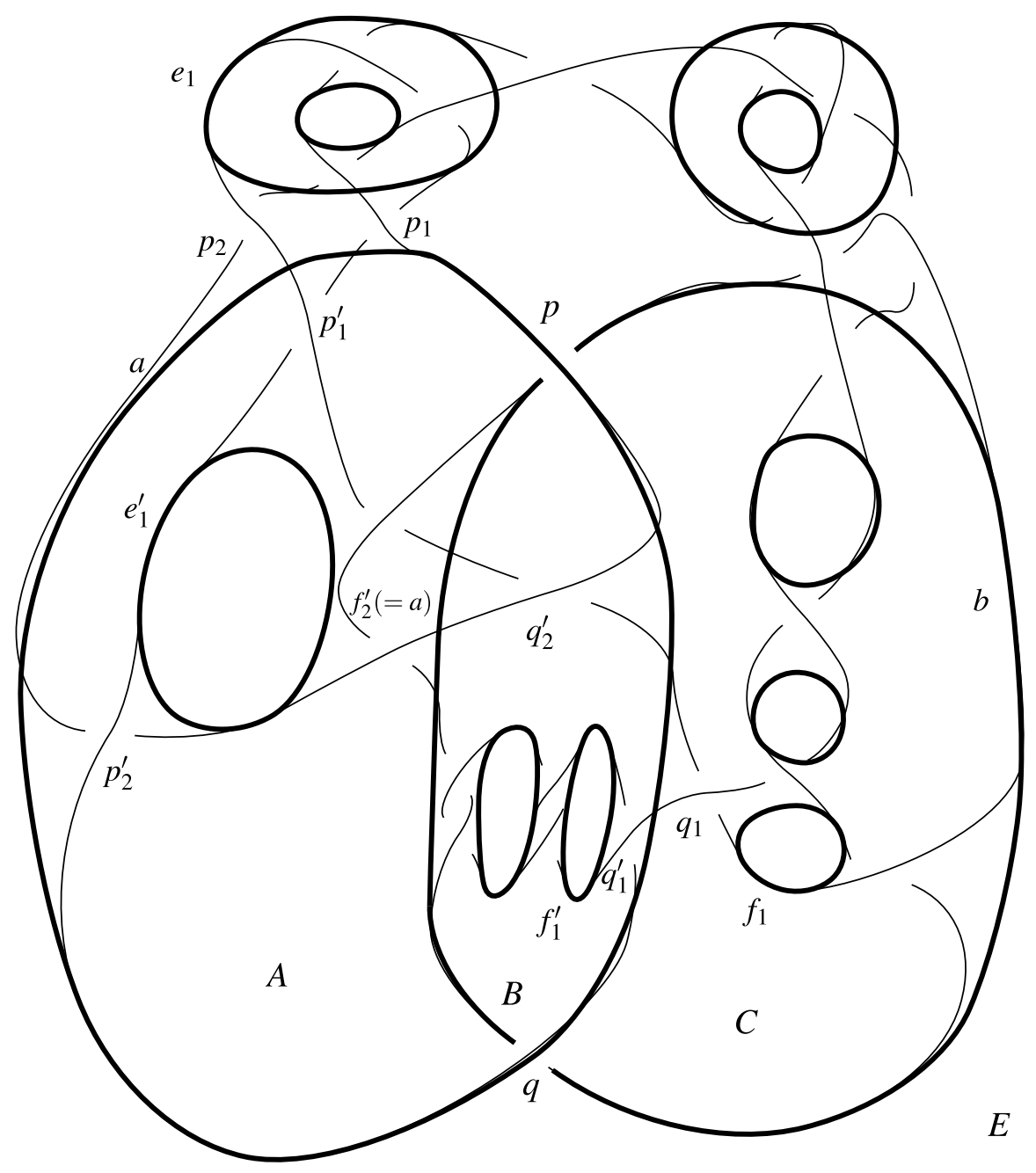

Figure 15. The link diagram in Figure 14 together with its Seifert circle picture, except that the crossings $p$ and $q$ are not smoothed out.

Now note that by this choice of skein resolution tree, each crossing is switched at most once, and only a positive crossing is switched in a nonterminal node $D_{+}$. Also, all overpasses of negative crossings are contained in $\beta$, so that in particular a terminal node contains a positive link (with a trivial split component). 
Since the contributions of the terminal nodes do not cancel, and are positive polynomials, we obtain the positivity of $\nabla$ on almost positive links, as proved in [13, Corollary 2.2].

To show now that max $\operatorname{deg} \nabla(L)=1-\chi(L)=-1-\chi(D)$, we must show that a terminal node $D_{t}$ has a non-zero contribution in degree $-1-\chi\left(D_{t}\right)$. This is the same as saying that some node $D_{t}^{\prime}$ in the tree has such a contribution in degree $-1-\chi\left(D_{t}^{\prime}\right)$, since $D_{t}$ will exist in the subtree for $D_{t}^{\prime}$. (Note that smoothing out a crossing brings in a factor $z$ from the skein relation of $\nabla$, which accords to the augmentation of $\chi$ by 1.)

We describe now how to find $D_{t}^{\prime}$. For this, we need to specify the path from $D$ to $D_{t}^{\prime}$ in the resolution tree. In other words, we need to specify a sequence of choices between a switch and a smoothing of crossings to extend $\beta$.

Let $x$ be the end of $\beta$ in orientation direction.

Assume $x$ is on $e$, and reaches a crossing $p_{t}$ in $E$, connecting it to the Seifert circle $e_{k}$. Let $e_{k}$ separate the plane into regions $S$ and $S^{\prime}$, and $S$ be the region containing $a$. Since $p_{k}$ is positive, we let (by maximal extension) $\beta$ pass over $p_{t}$ to $e_{k}$. Then we smooth out every non-nugatory crossing passed as undercrossing. If a crossing we reach as undercrossing is nugatory, then we switch it. (Note that the existence of certain crossings and their nugatority status are altered by applications of this rule to previous crossings.)

Optionally, we allow the move (22), where $r$ is nugatory. Now we argue that we can perform this procedure in a nice way.

LEMMA 4.1. After a sequence of switches/smoothings, and with an appropriate optional choice of moves (22) (and their reflections), $x$ can be made to reach the undercrossing of $p_{t}$, but not to leave $e_{k}$ or its interior $S^{\prime}$ through another crossing $r \neq p_{t}$ attached to $e_{k}$ on the same side $S$ as a.
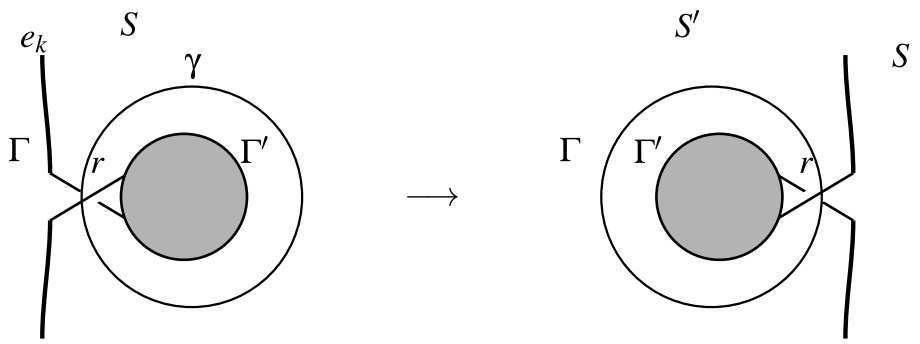

Proof. First, $r$ is reached as undercrossing from within $e_{k}$, so if $r$ is not nugatory, it is smoothed out. Now assume some of these crossings $r$ is nugatory. Then there is a loop $\gamma$ such that $D \cap \gamma=\{r\}$. Let $\Gamma$ be the region of $\gamma$ containing $e_{k}$. It obviously also must contain $a$, since $e_{k}$ is connected to $a$ by $p_{t}$, and $r \neq p_{t}$ by assumption. Then the effect would be the same as if we flip $r$ and the region $\Gamma^{\prime}=\mathbb{R}^{2} \backslash \Gamma$ into $e_{k}$, so that it is contained in $S^{\prime}$, as shown in (22). 
We have now ensured that $\beta$ does not leave $S^{\prime}$ until it reaches the undercrossing of $p_{t}$.

LEMMA 4.2. When $x$ reaches the undercrossing of $p_{t}$, all crossings $\beta$ passes within $e_{k}$ are nugatory.

Note that this lemma means in particular that $p_{t}$ becomes nugatory when passed by $x$ the second time (as undercrossing).

Proof of Lemma 4.2. Assume $\mu$ were a non-nugatory crossing $\beta$ passes. Then we argue that, by the way $\beta$ was chosen, $\mu$ is positive. To see this, note first that, since $\mu$ is positive in $D$, if $\mu$ were negative, it must have been switched, and hence passed by $x$. However, if it is non-nugatory after being passed by $x$, it is so before. Therefore, when $\mu$ is passed by $x$ the first time (i.e., one of the strands crossing at $\mu$ is passed by $x$ ), then by construction of $\beta$, if $\mu$ is underpassed by $x$, it would have been smoothed out. Thus it is overpassed, and not switched. If/when $\mu$ is passed by $x$ the second time, it is a self-crossing of $\beta$, and so not affected.

Note also that the part of $\beta$ between the two passes of $p_{t}$ is an unknotted, splittable arc, that is, turns into an unknotted, split component $\phi$ if $p_{t}$ would have been smoothed out. Since a positive diagram of the unknot has only nugatory crossings, all self-crossings of $\phi$ are nugatory. If such arc has a positive crossing with some other component $\psi$ in $S^{\prime}$, it would also have a negative one by the Jordan curve theorem, and because $l k(\phi, \psi)=0$. But any crossing between different components is non-nugatory, whereas by construction, all the negative crossings passed by $\beta$ are nugatory. This gives a contradiction.

Now, if $\beta$ passes a crossing $p_{t}^{\prime}$ connecting $e$ with some Seifert circle $e_{k}^{\prime}$ in $A$, then if $p_{t}^{\prime}$ is not nugatory, smooth it out (since it is passed from below, when coming from $e$, thereby remaining with $x$ in $e \subset a$ ), or otherwise apply the same procedure as with the region $\Gamma^{\prime}$ before.

After passing $q$ with $x$, handle $f_{i}^{\prime}$ as the $e_{i}^{\prime}$ and $f_{i}$ as the $e_{i}$. In $B$ there may be crossings $f_{i}^{\prime}$ that connect $a$ and $b$, but these are "handled" by being smoothed out.

After all $f_{i}$ and $f_{i}^{\prime}$, we arrive with $x$ at the start of $\beta$, the overcrossing of $p$, and $\beta$ closes to a split loop. See Figure 16 .

All crossings on $\beta$ are nugatory except $p$ and $q$. Now if $D$ is of the form



then $L$ is split and $\nabla=0$. Otherwise the elements of at least one of the four pairs of Seifert circle segments $\left\{e, e^{\prime}\right\},\left\{e^{\prime}, f\right\},\left\{f, f^{\prime}\right\},\left\{f^{\prime}, e\right\}$ are connected to 


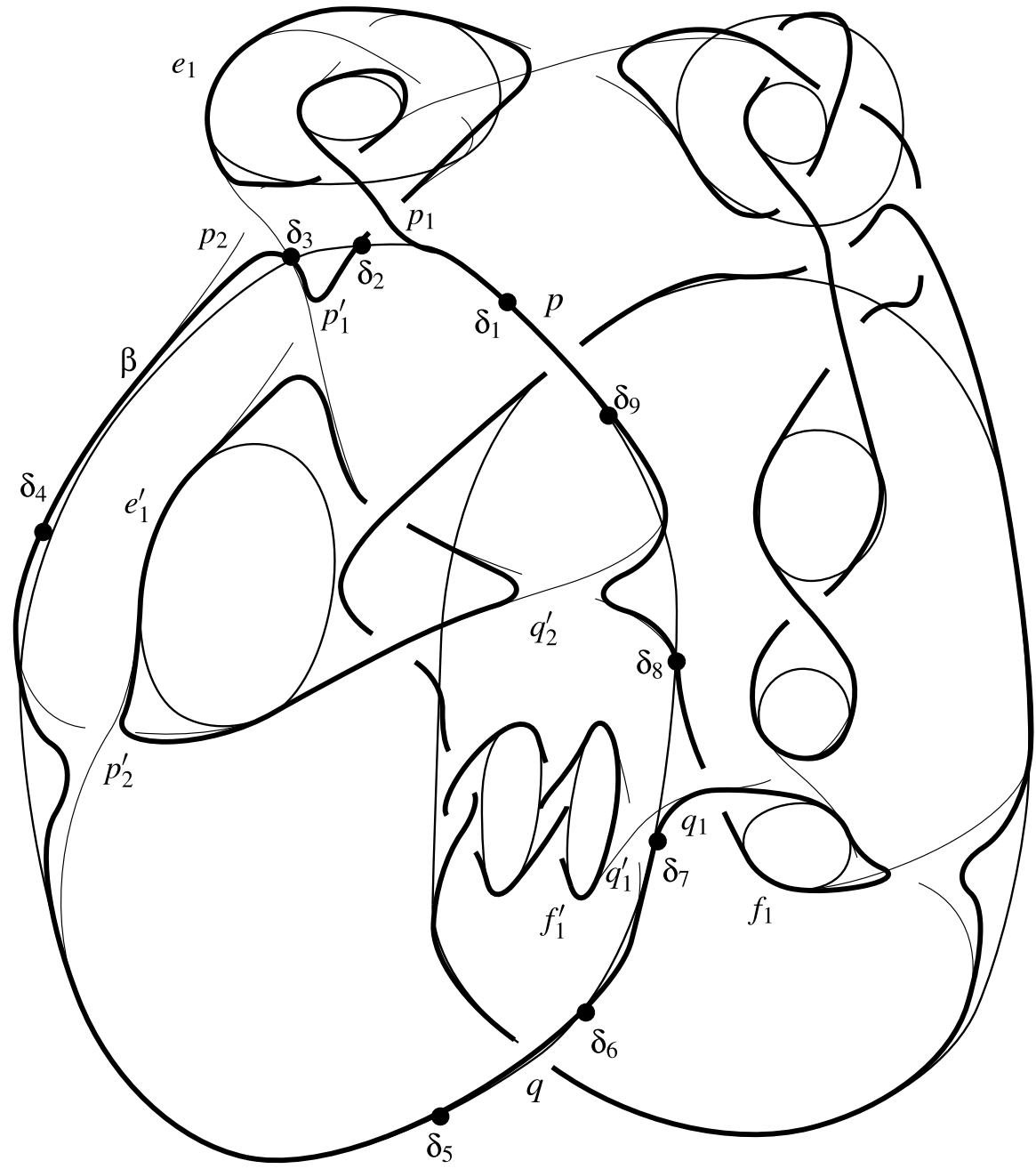

Figure 16. The diagram from Figure 15, but now with the result of the smoothings described in the proof of Theorem 1.5. The component that passes over the crossings $p$ and $q$ is now the curve $\beta$, and the intermediate points $\delta_{i}$ of $x$ are also indicated.

each other by a chain of crossings and (possible) intermediate Seifert circles. Let $\tau(D)$ be the number of such pairs. 
Now, mark on $e \cup f$ points $\delta_{1}, \ldots, \delta_{n}$ before and after passing $p$ and $q$, and between two crossings attached to $e$ or $f$. Start the indexing with the point $\delta_{1}$ after (the overpass of) $p$.

Let $\beta_{1}, \ldots, \beta_{n}$ be the intermediate parts of $\beta$ obtained as described above, when $x$ passes through $\delta_{1}, \ldots, \delta_{n}$, and $D_{1}, \ldots, D_{n}$ be the corresponding diagrams in the skein tree, so that $D=D_{1}$. Note that between $\delta_{i}$ and $\delta_{i+1}$, the bridge $\beta$ exits $e \cup f$ into one of the regions $A, B, C, E$ and returns on $e \cup f$ through the exiting crossing. Thus, by the previous lemmas, in all $D_{i}$ the crossings on $\beta$ are nugatory except $p$ and $q$ (if latter is on $\beta$ ). Also, since only one of $A, B, C, E$ is altered between $\delta_{i}$ and $\delta_{i+1}$, we have that $\tau\left(D_{i+1}\right)=\tau\left(D_{i}\right)$ or $\tau\left(D_{i+1}\right)=\tau\left(D_{i}\right)-1$. Now let $n^{\prime}$ be the maximal $i \in\{1, \ldots, n\}$, such that $\tau\left(D_{i}\right)>0$. Such $n^{\prime}$ exists, because in $D_{n}$ all crossings $\neq p, q$ on $\beta$ are nugatory, and $e \cup f \subset \beta$, so that $\tau\left(D_{n}\right)=0$, while $\tau(D)=\tau\left(D_{1}\right)>0$.

Then we set $D_{t}^{\prime}:=D_{n^{\prime}}$. This diagram $D_{t}^{\prime}$ is non-split by choice of $\beta$, since we never smoothed out nugatory crossings. Since $\tau\left(D_{t}^{\prime}\right)=1$, it is easy to see that $D_{t}^{\prime}$ can be turned into a positive non-split diagram $D_{t}^{\prime \prime}$ with $\chi\left(D_{t}^{\prime \prime}\right)=\chi\left(D_{t}^{\prime}\right)+2$. To achieve this, first remove all crossings on $\beta$ different from $p$ and $q$ (as they are nugatory), and then apply a flype, and Reidemeister II move, annihilating $p$ and $q$. Then $\operatorname{deg} \nabla\left(D_{t}^{\prime}\right)=-1-\chi\left(D_{t}^{\prime}\right)$, and thus $D_{t}^{\prime}$ has a non-trivial contribution in degree $-1-\chi\left(D_{t}^{\prime}\right)$, with which we are done.

The following is easy to see directly, but also worth mentioning as a consequence of our proof.

Corollary 4.1. Assume a link $L$ has an almost positive non-split diagram that is not of the form (23) (where the four encircled tangles may contain only positive crossings). Then $L$ is non-split.

REMARK 4.1. The non-cancellation argument in the skein resolution tree seems essential, and also explains why the equality in Theorem 1.5 cannot be proved in this way for other classes. Indeed, for example it is not always true for almost alternating links. However, Corollary 4.1 holds in an analogous form for almost alternating link diagrams, and was proved by Tsukamoto [81].

As a consequence using Theorem 5 of [71], we have the following corollary.

COROllary 4.2. If $L$ is an almost positive non-split link, then $\max \operatorname{deg} \Delta_{L}=\min \operatorname{deg} V_{L}$.

This solves Conjecture 5.2 of [70], which stated this property for almost positive knots. Using the work therein, we obtain:

COROLLARY 4.3. There are only finitely many almost positive knots with the same Alexander polynomial. 
4.2. General properties of examples. Almost positive knots seem a much smaller class than almost alternating ones. (For example, the property of all Montesinos links to be alternating or almost alternating, which we conveniently used several times before in the proofs, does not hold for almost positive links.) Since there are equally many almost positive and almost alternating diagrams (up to mirroring) of given crossing number, this suggests that in general almost alternating diagrams admit much more drastic crossing number reductions. We also realized that an almost alternating knot has infinitely many almost alternating diagrams, while from [70] we know an almost positive knot has only finitely many (reduced) almost positive diagrams.

The examples proving Theorem 1.4 are much more complicated to obtain, in that consideration of diagrams of genus $g=2$ is no longer sufficient. (The reason will be explained in the end of this section.) In addition, the instrumentarium of applicable (criteria of) invariants is considerably restricted, since almost positive links inherit many properties of invariants of positive links, as we proved in [70], [71] and in Section 4.1. Relevant to our context, we know already for theoretical reasons that Morton's inequality (17), and the condition max $\operatorname{deg} \Delta=g$ cannot be used anymore (in the way we did it for almost alternating knots).

In practice, the method of calculating $\mathcal{V}_{n}$ of (20) fails, too, for genus $g=3$. In that case, Theorem 2.10 renders $\mathcal{V}_{n}$ beyond the scope of any even moderately reasonable computability for $n \geq 5$. For $n \leq 3$, we observed in [73] that $\mathcal{V}_{n}$ gives almost entirely trivial information, because of the very special type of the evaluations of $V$ it captures [42], [37]. Trying to translate the calculation of Section 9 of [73] to genus 3, we found enough $V_{4}$-values in genus 3 diagrams to cover $V_{4}(K)$ for any of the more than 100,000 prime non-alternating $\leq 16$ crossing knots $K$ with $\max \operatorname{deg}_{m} P(K) \leq 6$. This suggests the $V_{4}$-test to be of little relevance either. On our examples (explained and given below), we attempted the stronger skein polynomial congruence test for $n=4$, as explained in $[73, \S 9]$. Then the same outcome was observed.

This explains why new tools are necessary. We will again use information completely obtainable from the Jones polynomial, but in a very different way. Still our methods succeed only at some cost. Beside the increase in algorithmical and computational effort, they also, unfortunately, do not permit us to decide on further properties. In particular, we do not know whether these examples have or have not at all minimal genus diagrams, thereby leaving open Question 4.1. Also, we cannot control easily their Alexander polynomials, or construct infinitely many. Remember we know from Corollary 4.3 that an analogue of Theorem 1.3 is no longer true.

Before we turn to rigorous arguments, let us mention that our results lend further interest to the following question. 
Question 4.2. Assume an almost positive knot has maxcf $\Delta=1$. Is it (always) fibered? (Cromwell [13, Corollary 2.2] proved that $\max \operatorname{cf} \Delta>0$.)

The answer is in general firmly negative for almost alternating knots, as we showed. But we know from [71] that it is positive if an almost positive knot has an almost positive diagram of minimal genus. Such knots are much more common than their almost alternating counterparts. Still now we know that they are not all. On the opposite hand-side, all our examples for Theorem 1.4 have max cf $\Delta>1$.

4.3. The Vassiliev invariant test. The first, theoretically secure, but not most efficient tool is provided by the Gauß diagram formulas.

LEMMA 4.3. Let $D$ be an almost positive diagram with negative crossing $p$, and $x$ a positive crossing. Let $D^{\prime}$ be obtained from $D$ by a $\bar{t}_{2}^{\prime}$ twist applied at $x$. Then $v_{2}\left(D^{\prime}\right) \geq v_{2}(D)$ and $v_{3}\left(D^{\prime}\right) \geq v_{3}(D)$. Moreover, in both inequalities, equality holds if and only if there is a crossing $x^{\prime} \simeq p$, such that the only crossings $x$ is linked with are $x^{\prime}$ and $p$.

Proof. Assume without loss of generality that $D$ is prime. (Since $v_{2}$ and $v_{3}$ are additive under connected sum, the composite case easily reduces to the prime one.) Assume first that a crossing $x^{\prime}$ exists such that $x^{\prime} \simeq p$, and $x^{\prime}$ and $p$ are the only crossings linked with $x$. Then we have a Gauß diagram like this:
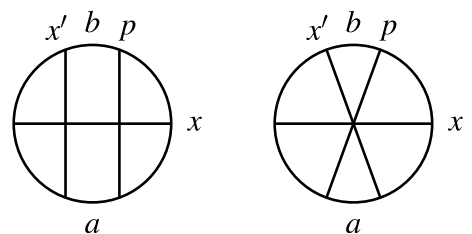

The diagram $D$ is prime, and $x^{\prime} \simeq p$ means that no chord intersects one of $x^{\prime}$ and $p$ but not the other. This implies that there are no basepoints of chords on the segments $a$ and $b$. Then in the knot diagram, $x^{\prime}$ and $p$ form a trivial clasp. Resolving that clasp renders $x$ nugatory, which shows that in fact twists at $x$ in $D$ are isotopies, whence $v_{2}\left(D^{\prime}\right)=v_{2}(D)$ and $v_{3}\left(D^{\prime}\right)=v_{3}(D)$.

Now assume the opposite situation, that is, that no such $x^{\prime}$ exists. We will show the increase of $v_{2}$ and $v_{3}$ as follows. We will argue that among the 'new' matching configurations in (14) and (15), that is, those involving a crossing created by the twist, the positive ones outnumber the negative ones. In the case of $v_{3}$, this is done by assigning to each new negative configuration an 'equilibrating' (new) positive one. We need to pay attention that each positive new configuration gets assigned at most once, and to observe that at least one remains unassigned.

Lemma 2.3 easily implies that if $x$ is linked with only two crossings, and $p$ is one of them, then the other crossing $x^{\prime} \simeq p$. With the argument, we can 
assume that $x$ is linked with (a) only positive crossings, or (b) with $p$ and at least 3 other crossings. Let $x_{i}$ for $i \in\{0,1\}$ be the two new crossings added by the twist at $x$.

In case (a) a twist at $x$ creates only positive linked pairs for $v_{2}$, and at least one of them is counted in one of the terms on the right of (14). Thus, $v_{2}$ is augmented.

As for $v_{3}$, let us trace what negative configurations the twist creates. No negative $(3,3)$-configuration is created, since by assumption $p \not x x$ (and hence also $p \not\left(x_{i}\right)$. Moreover, with one negative crossing in the diagram, no negative linked pair exists (with the weight in (15)). The only new negative configurations created may be of type $(4,2) 0$. Each such configuration $\left(x_{i}, p, q\right)$ is made up of a chord $q \cap x$ and $x_{i}, i \in\{0,1\}$ being one of the two new positive crossings created by the twist. Observe that only one of the $x_{i}$ occurs in such configuration because of the orientation of arrows. Without loss of generality, let this be $x_{1}$.

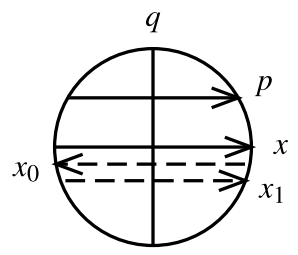

Then $\left(x_{1}, q\right)$ is a positive new linked pair which we make equilibrate this negative $(4,2) 0$ configuration. Note that this way each $\left(x_{1}, q\right)$ is assigned to at most one negative $(4,2) 0$ configuration $\left(x_{1}, q, p\right)$.

Since $\left(x, x_{1}, q\right)$ is a new (and unassigned) positive $(4,2) 0$ for each $q \cap x$ (with the arrow orientations as in the diagram above), $v_{3}$ increases.

In case (b), for $v_{2}$, the twist at $x$ creates at least 6 new positive linked pairs and two negative ones. After choosing the basepoint in (14) near some endpoint of $x$, we see that exactly one half of the groups of new linked pairs of either sign contribute to the right sum in (14). Thus, $v_{2}$ increases.

Regarding $v_{3}$, we first note that $p \cap x$ implies $p \cap x_{0,1}$. There are now new negative configurations of type $(3,3)$ and $(4,2) 0$. (As before, a negative linked pair in (15) would require two negative crossings.)

Consider first new negative configurations of type $(4,2) 0$. There are two types.

The first type are of the form $\left(p, x_{i}, q\right)$, such that $q \cap p$ and $q \not x_{i}$. (The latter property is equivalent to $q=x$ or $q \not x$.) By Lemma 2.3, there is a $p^{\prime}$ 
with $\left(p^{\prime}, x_{i}, q\right) \in(4,2) 0$ equilibrating $\left(p, x_{i}, q\right)$.
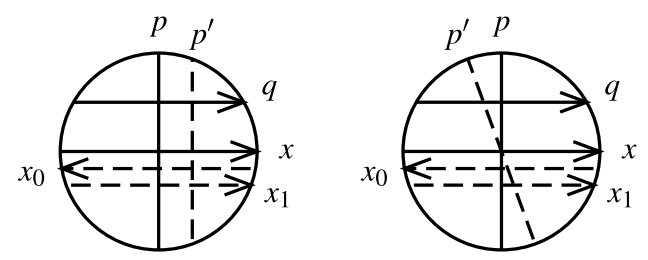

Any $\left(p^{\prime}, x_{i}, q\right)$ equilibrates at most one $\left(p, x_{i}, q\right)$, since one can regain $\left(p, x_{i}, q\right)$ from $\left(p^{\prime}, x_{i}, q\right)$ in a unique way: discard the arrow $p^{\prime}$ intersecting both other arrows, and put $p$ instead.

The other type of negative $(4,2) 0$ configurations are $\left(p, x_{i}, q\right)$ with $q \cap x$ and $q \backslash p$, such that $(p, x, q)$ is a $(4,2) 0$. These are equilibrated by the positive linked pair $\left(x_{i}, q\right)$.

Consider now new negative configurations of type $(3,3)$. For each $q \cap x$ (and $\left.q \cap x_{i}\right)$ giving a negative configuration $\left(p, q, x_{i}\right) \in(3,3)$, we have the positive linked pair $\left(q, x_{i}\right)$ equilibrating it (the linked pairs $\left(p, x_{i}\right)$ are neutral).

Now there are at least three positive arrows $p^{\prime}$ with $p^{\prime} \cap x$. For each such $p^{\prime}$, the triple $\left(x_{1}, x, p^{\prime}\right) \in(4,2) 0$ is positive. At most one of these at least three $(4,2) 0$ configurations is used to equilibrate a negative configuration $\left(x_{1}, x, p\right)$ of type $(4,2) 0$. Thus two positive contributions remain unassigned. Therefore, $v_{3}$ increases.

Definition 4.1. For a generator $K$, let

$$
\mathcal{I}_{K}:=\{0\} \cup\left\{i=1, \ldots, t(K): t_{i}(K)=1\right\}
$$

be the set of admissible groups of negative crossings of positive or almost positive diagrams in the series of $K$. (We exclude almost positive diagrams that have trivial reverse clasp after flypes.) Let us include positive diagrams into this scheme, meeting the convention that for such diagrams we set the group to 0 .

Proof of Theorem 1.4. We consider a positive or almost positive diagram $D$.

Now take a generator $K$ of genus $g$, and let $l=t(K)$. We first specify the minimal values of the $x_{i}$ in the representation $D=K\left(x_{1}, \ldots, x_{l}\right)$. Let $i_{0} \in \mathcal{I}_{K}$, and $p=p\left(K, i_{0}, 1\right)$ be the negative crossing of $D$ if $i_{0} \neq 0$. Set for $i=1, \ldots, l$

$$
s_{i}= \begin{cases}0 & i=i_{0}, \\ 2 & \text { if } t_{i}(K)=1 \text { and } p(K, i, 1) \approx p \\ 1 & \text { otherwise. }\end{cases}
$$

Notice that if $p \approx q$ and $r \sim q$, then one of $p$ and $r$ is equal to $q$. This means that

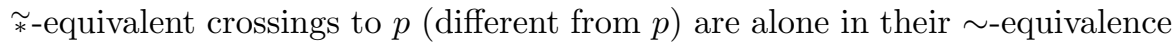
class. 
For $i_{0} \neq 0$ the diagram $D=K\left(x_{1}, \ldots, x_{l}\right)$ is almost positive, and it has no trivial (parallel) clasp after flypes for $x_{i_{0}}=s_{i_{0}}=0$ and $x_{i} \geq s_{i}$ when $i \neq i_{0}$. In order to discard uninteresting cases, we impose these conditions on the $x_{i}$ in this proof. If $i_{0}=0$, then $D$ is positive. Let us meet the convention that if $i_{0}=0$, any condition on $x_{i_{0}}$ is understood (also for the rest of the paper) to be empty, and the definition of $s_{i}$ in (24) is understood so that all $s_{i}=1$ (that is, we demand $x_{i} \geq 1$ for all $i=1, \ldots, l$ ).

Then any almost positive (resp. positive) diagram $D$ of an almost positive (resp. positive) knot $K_{0}$ of genus $g$ can be written as $K\left(x_{1}, \ldots, x_{l}\right)$, where $0 \neq i_{0} \in \mathcal{I}_{K}$ (resp. $i_{0}=0$ ) fixed, $x_{i_{0}}=0$ and $x_{i} \geq s_{i}$ for $i \neq i_{0}$, and $K$ is some generator of genus $g$ or $g+1$ (resp. only genus $g$ ).

Since on any such diagram, any $\bar{t}_{2}^{\prime}$ move at a positive crossing strictly augments $v_{2}$ and $v_{3}$, there are only finitely many diagrams that have given $v_{2}$ and $v_{3}$. Similarly, one proceeds for positive diagrams. It is easy to implement an algorithm that performs this twisting and uses (14) and (15) to calculate $v_{2}$ and $v_{3}$ on each diagram. Thus, knowing the list of generators, one can obtain a complete list of prime positive and almost positive diagrams of genus $g$ and given $v_{2}$ and $v_{3}$.

Consider the (prime) knot !12 1930 of [34]. It is almost positive (see [71, Corollary 8]), of genus 2. The above procedure was applied with $v_{2}=11$ and $v_{3}=35$ on generators of genus 2 and 3 , and then diagrams with $v_{2}$ and $v_{3}$ matching were tested for coincidence of the Jones polynomial with !12 1930 . The result was that ! $12_{1930}$ has only its obvious two almost positive diagrams, the 12 crossing table diagram (see Figure 8 of [68]), and the 13-crossing $(3,3,3,3,-1)$-pretzel diagram, both of genus 2 . This means that !12 1930 has no almost positive diagram of genus 3 , leading to the proof of the second part of Theorem 1.4.

For the first claim in Theorem 1.4, consider the following 17 crossing knot, shown on Figure 17. (We verified its crossing number by checking in the tables of [34] that it does not share its skein polynomial with any $\leq 16$ crossing prime knot.)

For this knot $g=\min \operatorname{deg} V=3$ is easily found using [71, Theorem 5]. One calculates $v_{2}=17$ and $v_{3}=66$, and can apply the same procedure as for $! 12_{1930}$ on genus 3 generators. Again only prime generators suffice, since the knot is prime. This can be seen most easily by calculation of its hyperbolic volume $(\approx 28.062)$. However, the test is much slower now, since $v_{2}$ and $v_{3}$ are higher, more twists are necessary to attain them, and accordingly more options exist to distribute these twists among the different $~$-equivalence classes. We must also consider positive diagrams, since we do not know a priori that the knot is not positive. But obviously, Lemma 4.3 holds for positive diagrams, too.

There is a way to accelerate the verification procedure. In fact, this optimization was crucially needed to find the 17 crossing example (and 143 other 




FiguRE 17. An almost positive knot with no almost positive minimal genus diagram.

ones), which in contrast to !12 1930 is not simple and not easy to arrive at. The idea is useful also for several later applications, and thus we explain it now.

4.4. Regularization of generators. We call this method regularization. The restrictions to crossing numbers of almost positive diagrams one can obtain from it are much stronger than those from $v_{2}$ and $v_{3}$, but they do not apply universally, in that they require certain initial conditions that must be checked case-by-case. We will explain the procedure for $\max \operatorname{deg} V$, which was applied in our situation, and then indicate possible refinements and generalizations.

Fix a generator $K$ and $i_{0} \in \mathcal{I}_{K}$ a priori. Let $l=t(K)$ and $D=K\left(x_{1}, \ldots, x_{l}\right)$ be a positive or almost positive diagram with negative crossing $p=p\left(K, i_{0}, 1\right)$ (that is, $x_{i_{0}}=0$ and all other $x_{i}>0$ ). Call $D$ genus-preserving if $D$ has minimal genus, and genus-reducing otherwise. Remark that which of either occurs can also be tested from the Jones polynomial, since from [71, Theorem 5] we know that for an almost positive knot $K, \min \operatorname{deg} V(K)=g(K)$. It can, of course, also be tested using Lemma 3.1 and Lemma 2.4.

Definition 4.2. Define the vector $\left(x_{1}, \ldots, x_{l}\right)^{\wedge}=\left(\hat{x}_{1}, \ldots, \hat{x}_{l}\right)$ by $\hat{x}_{i}=$ $\min \left(x_{i}, 2\right)$. For $D=K\left(x_{1}, \ldots, x_{l}\right)$ let $\hat{D}=K\left(\hat{x}_{1}, \ldots, \hat{x}_{l}\right)$.

Definition 4.3. A generator $K$ is called weakly regular if it satisfies the following property.

Consider a positive or almost positive diagram $D_{0}=K\left(x_{1}, \ldots, x_{l}\right)$ with $l=$ $t(K)$ and $D_{0}=\hat{D}_{0}$ (that is, $x_{i_{0}}=0$ for some $i_{0} \in \mathcal{I}_{K}$, and all other $x_{i} \in\{1,2\}$ ). 
Then for each such $D_{0}$ and each $j=1, \ldots, l$ with $x_{j}=2$ we have (25)

$\max \operatorname{deg} V\left(K\left(x_{1}, \ldots, x_{j}-1, \ldots, x_{l}\right)\right) \leq \max \operatorname{deg} V\left(K\left(x_{1}, \ldots, x_{j}, \ldots, x_{l}\right)\right)$.

LEMMA 4.4. If $K$ is weakly regular, then for each positive or almost positive diagram $D \in\langle K\rangle$ in the series of $K$ we have

$$
\max \operatorname{deg} V(D) \leq \max \operatorname{deg} V(\hat{D})-c(\hat{D})+c(D)
$$

Proof. If $D=\hat{D}$, then there is nothing to prove. Otherwise use induction on $c(D)$. Since $D=K\left(x_{1}, \ldots, x_{l}\right) \neq \hat{D}$, some $x_{i_{0}}>2$. Let $D_{1}=K\left(x_{1}, \ldots, x_{i_{0}}-1\right.$, $\left.\ldots, x_{l}\right)$ and $D_{2}=K\left(x_{1}, \ldots, x_{i_{0}}-2, \ldots, x_{l}\right)$. Then the skein relation for $V$ easily implies

$$
V(D)=\left(t^{2}+1\right) V\left(D_{1}\right)-t^{2} V\left(D_{2}\right)
$$

We always have $\hat{D}_{1}=\hat{D}$. If now also $\hat{D}_{2}=\hat{D}$ (i.e., $x_{i_{0}}>3$ ), then the claim easily follows by induction. Otherwise let $x_{i_{0}}=3$.

Since $c\left(D_{1,2}\right)<c(D)$, by induction assumption

$$
\begin{aligned}
& \max \operatorname{deg} V\left(D_{2}\right) \leq c\left(D_{2}\right)-c\left(\hat{D}_{2}\right)+\max \operatorname{deg} V\left(\hat{D}_{2}\right), \\
& \max \operatorname{deg} V\left(D_{1}\right) \leq c\left(D_{1}\right)-c\left(\hat{D}_{1}\right)+\max \operatorname{deg} V\left(\hat{D}_{1}\right) .
\end{aligned}
$$

Now

$$
c\left(D_{1}\right)-c\left(\hat{D}_{1}\right)=c\left(D_{2}\right)-c\left(\hat{D}_{2}\right)=2 \sum_{i \neq i_{0}: x_{i}>2}\left(x_{i}-2\right)=\sum_{i \neq i_{0}: t_{i}>4}\left(t_{i}-4\right) .
$$

Also

$$
\max \operatorname{deg} V\left(\hat{D}_{2}\right) \leq \max \operatorname{deg} V\left(\hat{D}_{1}\right),
$$

by applying $(25)$ to $j=i_{0}$ and $x_{j}=x_{i_{0}}-1$. Thus by taking (26) for $D_{1}$ (instead of $D$ ), and using $\hat{D}=\hat{D}_{1}$, we see that for both $i=1,2$,

$$
\max \operatorname{deg} V\left(D_{i}\right) \leq \max \operatorname{deg} V(\hat{D})+c\left(D_{1}\right)-c(\hat{D}) .
$$

Then from (27)

$$
\begin{aligned}
\max \operatorname{deg} V(D) & \leq 2+\max \operatorname{deg} V(\hat{D})+c\left(D_{1}\right)-c(\hat{D}) \\
& =c(D)-c(\hat{D})+\max \operatorname{deg} V(\hat{D})
\end{aligned}
$$

as claimed.

Now consider a diagram $D=K\left(x_{1}, \ldots, x_{l}\right)$ with $l=t(K)$ and $x_{i_{0}}=0$ (or $i_{0}=0$ ) which is genus-preserving, that is, min $\operatorname{deg} V(D)=g(D)$. By Lemma 3.1, this is equivalent to the property that either $D$ is positive, or that the negative crossing $p=p\left(D, i_{0}, 1\right)$ has no Seifert equivalent one. This means that for $i=1, \ldots, l$ with $i \neq i_{0}$ we have

$$
x_{i} \geq x_{i, \min }:= \begin{cases}2 & \text { if } t_{i}(D)=1 \text { and } i_{0} \neq 0 \text { and } p(D, i, 1) \sim_{S} p \\ 1 & \text { otherwise }\end{cases}
$$


Notice that if $x \sim q$ and $x \sim_{S} r$, and $q, r \neq x$, then $q=r$. This means that (for $i_{0} \neq 0$ ) Seifert equivalent crossings to $p$ are alone in their $\sim$-equivalence class (unless they are in the one of $p$, which we excluded a priori by genus minimality). Furthermore, we remark that $D$ is genus-preserving iff $\hat{D}$ is so.

DEFinition 4.4. Assume that $K$ is weakly regular and additionally that (25) is a strict inequality (i.e., not an equality) when $D=K\left(x_{1}, \ldots, x_{j}, \ldots, x_{l}\right)$ is genus-preserving. Then we call $K$ regular. Otherwise $K$ is irregular.

LEMMA 4.5. If $K$ is regular, then on any genus-preserving positive or almost positive diagram $D$ in the sequence of $K$ the inequality (26) is sharp (i.e., an equality).

Proof. If $D=\hat{D}$, the claim is trivial. Otherwise use induction on $c(D)$.

Since $D=K\left(x_{1}, \ldots, x_{l}\right) \neq \hat{D}$, some $x_{i_{0}}>2$. Let $D_{1}=K\left(x_{1}, \ldots, x_{i_{0}}-\right.$ $\left.1, \ldots, x_{l}\right)$ and $D_{2}=K\left(x_{1}, \ldots, x_{i_{0}}-2, \ldots, x_{l}\right)$. Apply $(27)$. Again $\hat{D}_{1}=\hat{D}$, and if $\hat{D}_{2}=\hat{D}$ (that is, $x_{i_{0}}>3$ ), we are easily done by induction. Otherwise let $x_{i_{0}}=3$.

Again (28) and (29) hold, but now since $\hat{D}_{1}=\hat{D}$ is genus-preserving,

$$
\max \operatorname{deg} V\left(\hat{D}_{2}\right)<\max \operatorname{deg} V\left(\hat{D}_{1}\right)
$$

by assumption of the lemma. Also, (29) is sharp by induction assumption. Thus, max $\operatorname{deg} V\left(D_{2}\right)<\max \operatorname{deg} V\left(D_{1}\right)$. Then by $(27), \max \operatorname{deg} V(D)=2+$ $\max \operatorname{deg} V\left(D_{1}\right)$, as we wish.

An important consequence of Lemma 4.5 is the following corollary.

Corollary 4.4. Assume $K$ is a regular generator. Then $c\left(D_{0}\right)-$ $\max \operatorname{deg} V\left(D_{0}\right)$ is bounded (above) on positive and genus-preserving almost positive diagrams $D_{0}$ in the sequence of $K$.

Note that, since diagrams $D$ with $D=\hat{D}$ are finitely many (in the sequence of $K$ they are $2^{l}$ if $i_{0}=0$ and $2^{l-1}$ otherwise), the regularity conditions can be tested. If they are found fulfilled, one can also calculate

$$
m_{K}:=\max \left\{c\left(D_{0}\right)-\max \operatorname{deg} V\left(D_{0}\right): \begin{array}{c}
D_{0} \text { positive or genus-preserving } \\
\text { series of } K
\end{array}\right\},
$$

by evaluating the maximum only over (the finite number of) diagrams $D_{0}=$ $\hat{D}_{0}$.

The result of testing regularity was as follows:

Proposition 4.1. Only 126 of the 4017 prime genus 3 generators $K$ are irregular. They have at most $12 \sim$-equivalence classes. All other (regular) $K$ satisfy $m_{K} \leq 6$. 
Since most generators $K$ turn out regular, including those with the most $\sim$-equivalence classes, the effort can be significantly reduced (see below). Let us make a few remarks on variations of the regularization procedure, even if they did not come to practical use here.

Note that the regularity conditions can be tested and used not only for given generator $K$, but in fact for a given pair $\left(K, i_{0}\right)$ with $i_{0} \in \mathcal{I}_{K}$. Then we have analogous $m_{K, i_{0}}$, and $m_{K}=\max \left\{m_{K, i_{0}}: i_{0} \in \mathcal{I}_{K}\right\}$. (This equality can be extended to irregular $K$ or pairs $\left(K, i_{0}\right)$ by the convention that $m_{K}$ resp. $m_{K, i_{0}}$ is set to be $\infty$ in this case.) However, since pairs $\left(K, i_{0}\right)$ are much more than $K$, the required splitting of the tests leads to an economy only if many $K$ are irregular (or have large $m_{K}$ ).

The idea of "regularizing" genus $g$ generators is not restricted to $\max \operatorname{deg} V$ regularity. An analogue of (27) holds also for $P$, and we used it as (18) already in one of our previous proofs. Then so do analogues of Lemmas 4.4 and 4.5, when $\max \operatorname{deg} V$ is replaced by $\max \operatorname{deg}_{l} P$. However, it became apparent after a few tests that more generators become $\max \operatorname{deg}_{l} P$-irregular. There are more invariants to regularize with. For example, instead of $\max _{\operatorname{deg}} P$ one can take

$$
\max \operatorname{deg}_{l, \mathcal{O}} P:=\max _{k \in \mathcal{O}} \max _{\operatorname{deg}_{l}}[P]_{m^{k}}
$$

for any non-empty $\mathcal{O} \subset G=\{0,2, \ldots, 2 g\}$ (so that $\max \operatorname{deg}_{l} P=\max _{\operatorname{deg}_{l, G}} P$ ). Alternatively, one can use $\max \operatorname{deg}_{t} P_{S}(t)$ of substitutions $P_{S}(t):=P(t, S(t))$ for any $S \in \mathbb{C}\left[t^{\mathbb{Q}}\right]$. This includes the case of $\max \operatorname{deg} V$, since (6) expresses $V(i t)$ by $P_{S}(t)$ for an $S \in \mathbb{C} t^{1 / 2}+\mathbb{C} t^{-1 / 2}$.

For diagrams of a fixed number (more than one) of negative crossings still variants of such regularity tests remain theoretically valid. We apply such a test extensively in [75] (omitting the repetition of the analogous theoretical details). When some proper initial "regularity" conditions are assumed, Vassiliev invariant tests may also apply.

4.5. Selection and verification of examples. After the regularity test and calculation of $m_{K}$, the restriction to a stand-alone $v_{2}-v_{3}$-test is necessary only for a small portion of the generators. Otherwise we have an additional crossing number bound of $c\left(D_{0}\right)$ for a positive or almost positive diagram $D_{0}$ of a genus 3 knot $K_{0}$ in the series of $K$, given by

$$
c\left(D_{0}\right) \leq m_{K}+\max \operatorname{deg} V\left(K_{0}\right) .
$$

This bound is in general considerably sharper than the one coming from the $v_{2}-v_{3}$-test. For example, the 17 crossing knot in Figure 17 has $\max \operatorname{deg} V=16$, and since $m_{K} \leq 6$ for all regular $K$, in the series of regular generators, we are restricted to $\leq 22$ crossings. In contrast, we found diagrams with up to 40 crossings matching the $v_{2}$ and $v_{3}$ of this knot. (They were generated when the $v_{2}-v_{3}$-test was performed in isolation to verify the example after it was obtained.) 
In fact, in order to obtain the 17 crossing example (and the other 143), this reduction is very important. First, we generated some genus-reducing diagrams of $\leq 20$ crossings and genus 4 . (They were found using the generators of genus 4, which were compiled as described in [75].) Then we discarded diagrams that admit a crossing-number reducing wave move (see Figure 5). It is easy to see that such a move does not spoil almost positivity, unless the diagram becomes positive. If the reduced diagram is positive, or almost positive and genus-preserving, the candidate can be discarded. Otherwise we have a simpler diagram of the same candidate. Since one diagram per candidate suffices, the original diagram is not useful to maintain in either case. (For genus reducing diagrams of genus two the list of candidates became empty already after this step. This was expected, since we proved in [73] that almost positive knots of genus one do not exist.)

We applied then the tool knotfind to discard those knots that can be transformed (by some of the moves in knotfind's repertoire) into positive or almost positive genus 3 diagrams. This tool is used (in some enhanced form) in the compilation of knot tables by Hoste, Thistlethwaite, and Weeks [35], and is indirectly included in their program KnotScape [34]. It also brought the remaining diagrams to 'minimal' form in order to eliminate most of the duplications of the same knots. (Starting with genus reducing diagrams of genus 3, the list of diagrams remained empty after this step. This strongly suggests that genus two examples do not exist, and explains the necessity of considering genus 3 knots.)

Then we had about 5000 candidates to be tested against all possible prime positive and genus-preserving almost positive genus 3 diagrams. We calculated $V, v_{2}$ and $v_{3}$, and generated diagrams with matching $v_{2}$ and $v_{3}$. For regular generators $K$, we restricted ourselves additionally to the maximal crossing number (31) imposed by $m_{K}$. (Even if many more diagrams were necessary to consider for irregular generators, this was now feasible, due to the fact that at most $12 \sim$-equivalence classes occur.) We calculated the Jones polynomial of all diagrams and discarded candidates as soon as their Jones polynomial occurred. After testing all 4017 generators, 144 of the about 5000 candidates remained. The 17 crossing knot in Figure 17 is apparently the unique example among them of smallest crossing number.

Remark 4.2. The tool knotfind has several known bugs. Most notably, the moves applied during the reduction process occasionally change the knot type of the diagram. This problem occurred also several times in the present procedure. However, in our case it simply suffices to locate, by calculation of invariants, (the results of) these faulty reductions, and to discard them. Of course, this restricts the list of potential examples, but only insignificantly, and the subsequent success justifies this decision. Obviously, we cannot claim 
anyway that our list of examples is complete in whatever sense (see the remark just below). Thus, the compilation of candidates is only heuristically to motivate. It is the examination of their validity that has to be rigorous, and this verification does not use knotfind.

Years later after this calculation was originally performed, an attempt at verification by a similar, but not identical procedure, reproduced this 17 crossing example, along with adding 5 others (verifiably) of 17 crossings, and some more (apparently) 18 crossing ones. In this course, we found that the irregularities in the 126 generators of Proposition 4.1 can be completely eliminated (at the only cost of having $m_{K} \leq 7$ ) when one defines regularity by demanding that (25) is strict whenever $x_{j}=x_{j, \min }+1$ (with $x_{j, \min }$ from (30) and $j \neq i_{0}$ ).

4.6. Almost special alternating knots and a problem. The verification algorithm developed for the examples in Theorem 1.4 can of course be used in a broader context. The following is easy (recall Definition 2.3).

PROPOSITION 4.2. If $K$ is almost special alternating, then all almost special alternating diagrams of $K$ have minimal genus.

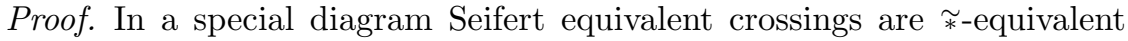
(see Lemma 2.4). Thus, from Lemma 3.1, a non-minimal genus almost special alternating diagram reduces to a special alternating one.

Clearly an almost special alternating knot is positive or almost positive. The converse is not always true, as follows from a condition of Murasugi on the signature $\sigma[52]$.

Corollary 4.5. If $K$ is almost special alternating, then $\sigma \geq 2 g-2$.

Proof. All almost special alternating diagrams of $K$ have minimal genus. Then from the work of [52] we have $\sigma=2 g$ in a special alternating diagram, and $\sigma$ changes by 0 or 2 under a crossing switch.

Many non-alternating positive and almost positive knots have $\sigma<2 g-2$, but none of them is very simple in crossing number. Among positive knots, we know meanwhile that all such knots have genus $g \geq 5$, except the knot $14_{45657}$ (with $g=\sigma=4$; see [75]). By Theorem 1.5, beside positive [13], also almost positive knots satisfy max $\operatorname{deg} \Delta=g$. Thus, we tested (more easily) $\sigma<2 \max \operatorname{deg} \Delta-2$, and then other direct-to-verify necessary conditions to be positive or almost positive. From the non-alternating prime knot tables of [34] up to 16 crossings we obtained 275 possible examples. (Most of them are indeed positive or almost positive, but we could not definitely verify it for all of them.)

The regularity and $v_{2}-v_{3}$-tests allow to find other examples of $g=3$.

Proposition 4.3. There are non-alternating, positive knots with $\sigma \geq 2 g-$ 2, which are not almost special alternating. 
Proof. One such knot is $13_{9684}$ (shown in [33], where another peculiar property of it was discovered). We explain how it was obtained.

We started with a list of non-alternating knots from the $\leq 16$ crossing prime knot tables of [34], that satisfy necessary conditions to be positive or almost positive of genus 3. By Proposition 4.2, to prove some of them not to be almost special alternating, only the 1867 prime special alternating generators of genus 3 need to be processed. In their series, only almost positive (and no positive) diagrams need to be considered. Knots with matching Jones polynomial in some of these diagrams were discarded.

Testing the other 2150 non-special generators (but now with positive diagrams included) then allows the identification of the positive or almost positive knots, and give reasonable evidence that the others are not. (Such evidence is accurate modulo the identification of knots from diagrams, see Remark 4.2, and the existence of examples as in Figure 17 of $\leq 16$ crossings.) There remained 69 knots, of which $13_{9684}$ has the fewest crossings. (This method also eliminated all potential genus 2 examples.) This knot $13_{9684}$ was later verified separately using the alone $v_{2}-v_{3}$-test on special generators (which took only about a minute!).

We know from [70] that for positive knots of $g \geq 2$ we have $\sigma \geq 4$, so none of the positive knots with $g=3$ has $\sigma<2 g-2$. (For example, $13_{9684}$ has $\sigma=2 g=6$.)

The real challenge was now to show that some of these examples are almost alternating. Clearly an almost special alternating knot is also almost alternating, since in [72] we proved that an alternating knot is not almost positive, and an alternating positive knot is special alternating. However, none of the examples we found (neither by the $\sigma$ condition, nor by the regularity and $v_{2}-v_{3}$-tests) could be proved to be almost alternating. (A similar outcome occurred in genus 4 . While the 1.4 million special generators, determined in [75], are too many to check, the first 9000 already sufficed to discredit all candidates.)

Question 4.3. If $K$ is positive or almost positive, and almost alternating, is then $K$ always almost special alternating? An equivalent formulation of this question is: if $K$ has almost positive and almost alternating (but no alternating) diagrams, does it also always have diagrams which are simultaneously both?

Note that in an almost positive almost alternating diagram either the dealternator is the negative crossing (and the diagram is almost special alternating), or both are the only 2 crossings in a block (as specified in Definition 2.4), and then cancel after a possible flype to a special alternating diagram.

We should in contrast stress that we could exclude only 10 of the 275 knots of $\sigma<2 g-2$ from being almost alternating, using the fact that they 
are not hyperbolic. Among the 69 examples with $\sigma \geq 2 g-2$, none could be excluded. In particular, the most conspicuous instances in both series, $13_{9684}$ and $14_{45657}$, remain undecided. Unfortunately, the lack of generally workable conditions on almost alternation makes the examination of the status even of single such examples more into a speculation.

Acknowledgments. The calculations were greatly assisted by the table access program KnotScape [34] and the graphic web interface Knotilus [24]. S. Rankin and M. Thistlethwaite provided some further technical support. Some of the work of this paper was presented at University of Waterloo, Canada, during a visit to L. Kauffman. I appreciate being given this opportunity for a talk. Part of the research was supported by Postdoc grant P04300 of the Japan Society for the Promotion of Science (JSPS) and by the National Research Foundation of Korea Grant funded by the Korean Government (NRF-2011-0027989). I am grateful to my JSPS host T. Kohno at the University of Tokyo for his encouragement. I thank the referee for his helpful comments.

\section{REFERENCES}

[1] T. Abe and K. Kishimoto, The dealternating number and the alternation number of a closed 3-braid, J. Knot Theory Ramifications 19 (2010), no. 9, 1157-1181. MR 2726563

[2] C. C. Adams, Toroidally alternating knots and links, Topology 33 (1994), no. 2, 353369. MR 1273788

[3] C. C. Adams, Das Knotenbuch, Spektrum Akademischer Verlag, Berlin, 1995. (The knot book, Freeman, New York, 1994.)

[4] C. C. Adams et al., Almost alternating links, Topology Appl. 46 (1992), 151-165. MR 1184114

[5] S. Akbulut and J. D. McCarthy, Casson's invariant for oriented 3-spheres, Mathematical Notes, vol. 36, Princeton, 1990. MR 1030042

[6] J. W. Alexander, Topological invariants of knots and links, Trans. Amer. Math. Soc. 30 (1928), 275-306. MR 1501429

[7] D. Bar-Natan, On the Vassiliev knot invariants, Topology 34 (1995), 423-472. MR 1318886

[8] D. Bennequin, Entrelacements et équations de Pfaff, Astérisque 107-108 (1983), 87161. MR 0753131

[9] M. Brittenham, Bounding canonical genus bounds volume, preprint, 1998; available at http://www . math.unl.edu/ mbrittenham2/personal/pprdescr.html.

[10] G. Burde and H. Zieschang, Knots, de Gruyter, Berlin, 1986. MR 0808776

[11] T. D. Cochran and R. E. Gompf, Applications of Donaldson's theorems to classical knot concordance, homology 3-spheres and property P, Topology 27 (1988), no. 4, 495-512. MR 0976591

[12] J. H. Conway, On enumeration of knots and links, Computational problems in abstract algebra (J. Leech, ed.), Pergamon, Elmsford, 1969, pp. 329-358. MR 0258014

[13] P. R. Cromwell, Homogeneous links, J. Lond. Math. Soc. (2) 39 (1989), 535-552. MR 1002465

[14] P. R. Cromwell, Knots and links, Cambridge University Press, Cambridge, 2004. MR 2107964 
[15] P. R. Cromwell and H. R. Morton, Positivity of knot polynomials on positive links, J. Knot Theory Ramifications 1 (1992), 203-206. MR 1164116

[16] R. Crowell, Genus of alternating link types, Ann. of Math. 69 (1959), no. 2, 258-275. MR 0099665

[17] R. Crowell, Nonalternating links, Illinois J. Math. 3 (1959), 101-120. MR 0099667

[18] C. H. Dowker and M. B. Thistlethwaite, Classification of knot projections, Topology Appl. 16 (1983), no. 1, 19-31. MR 0702617

[19] T. Fiedler, A small state sum for knots, Topology 32 (1993), no. 2, 281-294. MR 1217069

[20] T. Fiedler, Gauss sum invariants for knots and links, Mathematics and Its Applications, vol. 532, Kluwer Academic, Norwell, 2001.

[21] T. Fiedler and A. Stoimenow, New knot and link invariants, Proceedings of the international conference on knot theory "Knots in Hellas, 98", Series on Knots and Everything, vol. 24, World Scientific, Singapore, 2000. MR 1865701

[22] J. Franks and R. F. Williams, Braids and the Jones-Conway polynomial, Trans. Amer. Math. Soc. 303 (1987), 97-108. MR 0896009

[23] P. Freyd, J. Hoste, W. B. R. Lickorish, K. Millett, A. Ocneanu and D. Yetter, A new polynomial invariant of knots and links, Bull. Amer. Math. Soc. (N.S.) 12 (1985), 239-246. MR 0776477

[24] R. Furmaniak and S. Rankin, Knotilus, a knot visualization interface; available at http://knotilus.math.uwo.ca/.

[25] D. Gabai, The Murasugi sum is a natural geometric operation, Low-dimensional topology (San Francisco, Calif., 1981), Contemp. Math., vol. 20, Am. Math. Soc., Providence, 1983. MR 0718138

[26] D. Gabai, Foliations and genera of links, Topology 23 (1984), 381-394. MR 0780731

$[27]$ D. Gabai, The Murasugi sum is a natural geometric operation II, Combinatorial methods in topology and algebraic geometry (Rochester, N.Y., 1982), Contemp. Math., vol. 44, Am. Math. Soc., Providence, 1985, pp. 93-100. MR 0813105

[28] D. Gabai, Detecting fibred links in $S^{3}$, Comment. Math. Helv. 61 (1986), no. 4, 519555. MR 0870705

[29] D. Gabai, Foliations and the topology of 3-manifolds II, J. Differential Geom. 26 (1987), no. 3, 461-478. MR 0910017

[30] H. Goda, M. Hirasawa and R. Yamamoto, Almost alternating diagrams and fibered links in $S^{3}$, Proc. Lond. Math. Soc. 83 (2001), no. 2, 472-492. MR 1839463

[31] M. Hirasawa, The flat genus of links, Kobe J. Math. 12 (1995), no. 2, 155-159. MR 1391192

[32] M. Hirasawa, Triviality and splittability of special almost alternating links via canonical Seifert surfaces, Topology Appl. 102 (2000), no. 1, 89-100. MR 1739265

[33] M. Hirasawa and A. Stoimenow, Examples of knots without minimal string Bennequin surfaces, Asian J. Math. 7 (2003), no. 3, 435-446. MR 2129329

[34] J. Hoste and M. Thistlethwaite, KnotScape, a knot polynomial calculation and table access program; available at http://www.math.utk.edu/ morwen.

[35] J. Hoste, M. Thistlethwaite and J. Weeks, The first 1,701,936 knots, Math. Intelligencer 20 (1998), no. 4, 33-48. MR 1646740

[36] V. F. R. Jones, A polynomial invariant of knots and links via von Neumann algebras, Bull. Amer. Math. Soc. (N.S.) 12 (1985), 103-111. MR 0766964

[37] V. F. R. Jones, Hecke algebra representations of braid groups and link polynomials, Ann. of Math. 126 (1987), 335-388. MR 0908150

[38] T. Kanenobu, The augmentation subgroup of a pretzel link, Math. Sem. Notes Kobe Univ. 7 (1979), no. 2, 363-384. MR 0557309

[39] L. H. Kauffman, State models and the Jones polynomial, Topology 26 (1987), 395-407. MR 0899057 
[40] L. H. Kauffman, An invariant of regular isotopy, Trans. Amer. Math. Soc. 318 (1990), 417-471. MR 0958895

[41] R. Kirby and W. B. R. Lickorish, Prime knots and concordance, Math. Proc. Cambridge Philos. Soc. 86 (1979), no. 3, 437-441. MR 0542689

[42] W. B. R. Lickorish and K. C. Millett, Some evaluations of link polynomials, Comment. Math. Helv. 61 (1986), 349-359. MR 0860127

[43] W. B. R. Lickorish and K. C. Millett, A polynomial invariant for oriented links, Topology 26 (1987), no. 1, 107-141. MR 0880512

[44] W. B. R. Lickorish and M. B. Thistlethwaite, Some links with non-trivial polynomials and their crossing numbers, Comment. Math. Helv. 63 (1988), 527-539. MR 0966948

[45] P. M. Melvin and H. R. Morton, Fibred knots of genus 2 formed by plumbing Hopf bands, J. Lond. Math. Soc. (2) 34 (1986), no. 1, 159-168. MR 0859157

[46] W. W. Menasco, Closed incompressible surfaces in alternating knot and link complements, Topology 23 (1986), no. 1, 37-44. MR 0721450

[47] W. W. Menasco and M. B. Thistlethwaite, The Tait flyping conjecture, Bull. Amer. Math. Soc. (N.S.) 25 (1991), no. 2, 403-412. MR 1098346

[48] H. R. Morton, Infinitely many fibred knots having the same Alexander polynomial, Topology 17 (1978), no. 1, 101-104. MR 0486796

[49] H. R. Morton, Seifert circles and knot polynomials, Math. Proc. Cambridge Philos. Soc. 99 (1986), 107-109. MR 0809504

[50] K. Murasugi, On the genus of the alternating knot, J. Math. Soc. Japan 10 (1958), 94-105. 235-248. MR 0099664

[51] K. Murasugi, On a certain subgroup of the group of an alternating link, Amer. J. Math. 85 (1963), 544-550. MR 0157375

[52] K. Murasugi, On a certain numerical invariant of link types, Trans. Amer. Math. Soc. 117 (1965), 387-422. MR 0171275

[53] K. Murasugi, Jones polynomial and classical conjectures in knot theory, Topology 26 (1987), 187-194. MR 0895570

[54] K. Murasugi, On the braid index of alternating links, Trans. Amer. Math. Soc. 326 (1991), no. 1, 237-260. MR 1000333

[55] K. Murasugi and J. Przytycki, The skein polynomial of a planar star product of two links, Math. Proc. Cambridge Philos. Soc. 106 (1989), no. 2, 273-276. MR 1002540

[56] T. Nakamura, On canonical genus of fibered knot, J. Knot Theory Ramifications 11 (2002), no. 3, 341-352. MR 1905689

[57] M. Ozawa, Closed incompressible surfaces in complements of positive knots, Comment. Math. Helv. 77 (2002), 235-243. MR 1915040

[58] K. A. Perko, Invariants of 11 crossing knots, Publications Math. d'Orsay, 1980.

[59] M. Polyak and O. Viro, Gauss diagram formulas for Vassiliev invariants, Int. Math. Res. Not. 11 (1994), 445-454. MR 1316972

[60] M. Polyak and O. Viro, On the Casson knot invariant, Knots in Hellas'98, vol. 3; J. Knot Theory Ramifications 10 (2001), no. 5, 711-738. MR 1839698

[61] J. Przytycki and K. Taniyama, Almost positive links have negative signature, Abstr. Pap. Presented Am. Math. Soc. 12 (1991), no. 3, 327; J. Knot Theory Ramifications 19 (2010), no. 2, 187-289. MR 2647054

[62] D. Rolfsen, Knots and links, Publish or Perish, Berkeley, 1976. MR 0515288

[63] L. Rudolph, Positive links are strongly quasipositive, Proceedings of the Kirbyfest, Geometry and Topology Monographs, vol. 2, 1999, pp. 555-562. MR 1734423

[64] H. Seifert, Über das Geschlecht von Knoten, Math. Ann. 110 (1934), 571-592. MR 1512955

[65] A. Stoimenow, The signature of 2-almost positive knots, J. Knot Theory Ramifications 9 (2000), no. 6, 813-845. MR 1775388 
[66] A. Stoimenow, Gauß sum invariants, Vassiliev invariants and braiding sequences, J. Knot Theory Ramifications 9 (2000), no. 2, 221-269. MR 1749498

[67] A. Stoimenow, Knots of genus one, Proc. Amer. Math. Soc. 129 (2001), no. 7, 21412156. MR 1825928

[68] A. Stoimenow, Positive knots, closed braids, and the Jones polynomial, Ann. Sc. Norm. Super. Pisa Cl. Sci. 2 (2003), no. 2, 237-285. arXiv:math/9805078. MR 2004964

[69] A. Stoimenow, The crossing number and maximal bridge length of a knot diagram, Pacific J. Math. 210 (2003), no. 1, 189-199; with an appendix by M. Kidwell. MR 1989075

[70] A. Stoimenow, Gauss sums on almost positive knots, Compos. Math. 140 (2004), no. 1, 228-254. arXiv:math/9803073. MR 2004131

[71] A. Stoimenow, On polynomials and surfaces of variously positive links, J. Eur. Math. Soc. 7 (2005), no. 4, 477-509. arXiv:math/0202226. MR 2159224

[72] A. Stoimenow, On some restrictions to the values of the Jones polynomial, Indiana Univ. Math. J. 54 (2005), no. 2, 557-574. MR 2136821

[73] A. Stoimenow, Knots of (canonical) genus two, Fund. Math. 200 (2008), no. 1, 1-67. arXiv:math/0303012. MR 2443760

[74] A. Stoimenow, On the crossing number of semiadequate links, Forum Math. 26 (2014), no. 4, 1187-1246. MR 3228928

[75] A. Stoimenow, Diagram genus, generators and applications, Monographs and Research Notes in Mathematics, Chapman and Hall/CRC, Boca Raton, 2016.

[76] A. Stoimenow and A. Vdovina, Counting alternating knots by genus, Math. Ann. 333 (2005), 1-27. MR 2169826

[77] K. Taniyama, A partial order of knots, Tokyo J. Math. 12 (1989), no. 1, 205-229. MR 1001742

[78] M. B. Thistlethwaite, A spanning tree expansion for the Jones polynomial, Topology 26 (1987), 297-309. MR 0899051

[79] M. B. Thistlethwaite, On the Kauffman polynomial of an adequate link, Invent. Math. 93 (1988), no. 2, 285-296. MR 0948102

[80] P. Traczyk, Non-trivial negative links have positive signature, Manuscripta Math. 61 (1988), 279-284. MR 0949818

[81] T. Tsukamoto, A criterion for almost alternating links to be non-splittable, Math. Proc. Cambridge Philos. Soc. 137 (2004), no. 1, 109-133. MR 2075045

[82] Y. Yokota, Polynomial invariants of positive links, Topology 31 (1992), no. 4, 805-811. MR 1191382

[83] L. Zulli, The rank of the trip matrix of a positive knot diagram, J. Knot Theory Ramifications 6 (1997), no. 2, 299-301. MR 1452443

A. Stoimenow, Gwanguu Institute of Science and Technology, School of General Studies, GiSt College, 123 Cheomdan-gwagiro, Gwanguu 500-712, Korea

E-mail address: stoimeno@stoimenov.net

URL: http://stoimenov.net/stoimeno/homepage/ 